\title{
Title: Discovery of short-course anti-wolbachial quinazolines for elimination of filarial worm infections
}

Authors: Malina A. Bakowski ${ }^{1}$, Roohollah Kazem Shiroodi ${ }^{1}$, Renhe Liu ${ }^{2}$, Jason Olejniczak ${ }^{1}$, Baiyuan Yang ${ }^{1}$, Kerstin Gagaring ${ }^{1}$, Hui Guo ${ }^{1}$, Pamela M. White ${ }^{3}$, Laura Chappell ${ }^{3}$, Alain Debec $^{4}$, Frédéric Landmann ${ }^{5}$, Bettina Dubben ${ }^{6}$, Franziska Lenz ${ }^{6}$, Dominique Struever ${ }^{6}$, Alexandra Ehrens ${ }^{6}$, Stefan J. Frohberger ${ }^{6}$, Hanna Sjoberg ${ }^{7}$, Nicolas Pionnier ${ }^{7}$, Emma Murphy ${ }^{7}$, John Archer ${ }^{7}$, Andrew Steven 7 , Valerine C. Chunda ${ }^{8,9}$, Fanny F. Fombad ${ }^{8,9}$, Patrick W.

Chounna $^{8,9}$, Abdel J. Njouendou ${ }^{8,9}$, Haelly M. Metuge ${ }^{8}$, Bertrand L. Ndzeshang ${ }^{8,9}$, Narcisse V. Gandjui $^{8,9}$, Desmond N. Akumtoh ${ }^{8,9}$, Tayong D. B. Kwenti ${ }^{8,9}$, Ashley K. Woods ${ }^{1}$, Sean B. Joseph $^{1}$, Mitchell V. Hull ${ }^{1}$, Wen Xiong ${ }^{2}$, Kelli L. Kuhen ${ }^{1}$, Mark Taylor ${ }^{7}$, Samuel Wanji ${ }^{8,9}$, Joseph D Turner ${ }^{7}$, Marc P. Hübner ${ }^{6}$, Achim Hoerauf ${ }^{6}$, Jason Roland ${ }^{1}$, Matt S. Tremblay ${ }^{1}$, Peter G. Schultz ${ }^{1}$, William Sullivan ${ }^{3}, \mathrm{Xin}_{-} J i e \mathrm{Chu}^{2}$, H. Michael Petrassi ${ }^{1}$, Case W. McNamara ${ }^{1}$.

\section{Affiliations:}

${ }^{1}$ Calibr at Scripps Research, La Jolla, California 92037.

${ }^{2}$ The Global Health Drug Discovery Institute (GHDDI), Beijing, China.

${ }^{3}$ University of California Santa Cruz, Santa Cruz, California 95064.

${ }^{4}$ Jacques Monod Institute. University Paris Diderot / CNRS. 75013 Paris, France.

${ }^{5}$ CRBM, University of Montpellier, CNRS, Montpellier, France.

${ }^{6}$ Institute for Medical Microbiology, Immunology and Parasitology, University Hospital Bonn, Bonn, Germany

${ }^{7}$ Research Centre for Drugs and Diagnostics, Department of Parasitology, Liverpool School of Tropical Medicine, Pembroke Place, Liverpool, UK L3 5QA.

${ }^{8}$ Research Foundation in Tropical Diseases and the Environment, Buea, Cameroon. 
${ }^{9}$ Parasite and Vector Biology Research Unit, Department of Microbiology and Parasitology, Faculty of Science, University of Buea, Buea, Cameroon.

*Corresponding author.Email: cmcnamara@ scripps.edu (C.W.M).

\title{
Overline: Infectious disease
}

One Sentence Summary: This study identifies oral, highly efficacious anti-wolbachial compounds to cure filarial infections.

\begin{abstract}
:
Parasitic filarial nematodes cause debilitating infections in people in resource-limited countries. A clinically validated approach to eliminate worms uses a 4-6 week course of doxycycline that targets Wolbachia, a bacterial endosymbiont required for worm viability and reproduction. However, the prolonged length of therapy and contraindication in children and pregnant women have slowed adoption of this treatment. Here we describe discovery and optimization of quinazolines CBR417 and CBR490 that with a single dose achieve >99\% elimination of Wolbachia in the in vivo Litomosoides sigmodontis filarial infection model. The efficacious quinazoline series was identified by pairing a primary cell-based high content imaging screen with an orthogonal ex vivo validation assay to rapidly quantify Wolbachia elimination in Brugia pahangi filarial ovaries. We screened 300,368 small molecules in the primary assay and identified 288 potent and selective hits. Of 134 primary hits tested, only $23.9 \%$ were active in the worm-based validation assay, eight of which contained a quinazoline heterocycle core. Medicinal chemistry optimization generated quinazolines with excellent pharmacokinetic profiles in mice. Potent anti-wolbachial activity was confirmed in $L$. sigmodontis, B. malayi, and Onchocerca ochengi in vivo preclinical models of filarial disease, and in vitro selectivity against Loa loa (a safety concern in endemic areas). The favorable efficacy and in vitro safety profiles of CBR490 and CBR417 further support these as clinical candidates for treatment of filarial infections.
\end{abstract}

\section{Introduction}


Parasitic filarial nematodes, including ones that cause lymphatic filariasis and onchocerciasis (also known as river blindness), were estimated in 2013 to infect 43.8 million and 17 million people worldwide, respectively (1) with over a billion at risk of infection (2). Neither lymphatic filariasis nor onchocerciasis are commonly lethal; however, they are a recognized source of significant morbidity and suffering (3). In addition to acute symptoms, these long-term infections often result in disfigurement, social discrimination, and contribute to increased poverty of the afflicted individuals and their families. Both lymphatic filariasis and onchocerciasis are caused by long-lived filarial nematodes (roundworms) transmitted by bloodfeeding insect vectors. Onchocerciasis is caused exclusively by Onchocerca volvulus and lymphatic filariasis is caused mainly by Wuchereria bancrofti, and by the closely related Brugia species (B. malayi and B. timori). While the adults (macrofilariae) persist within human hosts for up to 15 years, they release thousands of microfilariae each day that are either the main cause or contribute to symptoms of disease and are also the developmental stage responsible for transmission back to the insect vector.

There is no short course cure for these infections and current control treatments have been centered on mass drug administration (MDA) campaigns to interrupt transmission and to alleviate symptoms for the duration of the reproductive life-span of adult female parasites, variably estimated at 5-8 years (4). The recommended treatment for onchocerciasis is the drug ivermectin (Mectizan), administered at least once yearly to all at risk of infection. Ivermectin works by killing microfilariae and temporarily sterilizing, but not killing, adult worms. Current recommended treatment for lymphatic filariasis varies by geography: albendazole together with ivermectin in Africa where onchocerciasis is co-endemic with lymphatic filariasis, and albendazole with diethylcarbamazine in the rest of the world. These treatments likewise lead to the death of microfilariae, not the adult parasites, and these drug regimens must be maintained for at least five years. Although MDA of ivermectin for onchocerciasis has been ongoing for over 25 years (5), there are concerns over development of drug resistance (6), which has already been reported in veterinary medicine $(7,8)$, the extensive MDA coverage that must be achieved to meet elimination targets $(9,10)$, and with overall compliance of at-risk populations $(11,12)$. Additionally, treatments with diethylcarbamazine or ivermectin are contraindicated in patients with a high load of microfilariae of the African eye worm Loa loa, $(>30,000-50,000$ microfilariae/mL of blood) due to severe adverse events (13). Lower densities of microfilariae 
can also cause other, non-neurological adverse events, and overall concern over the potential for L. loa-associated side effects can reduce adherence to MDA campaigns (12).

An attractive and clinically-validated strategy for developing a treatment to selectively kill adult worms is targeting the bacterial endosymbiont of onchocerciasis and lymphatic filariasis -causing worms, Wolbachia, which is absent from L. loa nematodes. Wolbachia are gram-negative obligate intracellular bacteria that are widely distributed among a variety of arthropods, where they are considered to be reproductive parasites, known for induction of parthenogenesis, feminization and male killing (14). In filarial nematodes, Wolbachia are essential endosymbionts, needed by adult worms for both reproduction and viability. Early experiments have shown that tetracycline treatment could prevent experimental infections of rodents with Brugia (15) and L. sigmodontis but not with the Wolbachia free species Acanthocheilonema viteae (16). The finding that Wolbachia is widely distributed among filarial nematodes (17), stimulated great interest in antibiotic anti-filarial therapy (18). Subsequently, it has been shown in humans that treatment with doxycycline over a period of four to six weeks to eliminate Wolbachia from adult worms is sterilizing and eventually macrofilaricidal, with life span of Wolbachia-depleted worms reduced by 70-80\% (from 10 years to 2-3 years) (19-22). An added benefit of this approach is potential reduction of inflammation, as adverse inflammatory reactions to anthelmintic treatment have been associated with Wolbachia released in patient plasma $(23,24)$. However, doxycycline is contraindicated for treatment of pregnant women and children under eight years of age. The prolonged length of treatment also represents potential challenges with compliance and contributes to cost of therapy, highlighting the need for faster, safer, and more effective therapies. Here we describe the identification of quinazolines CBR417 and CBR490 that are able to achieve very rapid clearance of Wolbachia from filarial nematodes in in vivo preclinical models of disease, and which offer the potential for development of a short-course cure to treat filarial worm infections.

\section{Results}

Primary high-throughput phenotypic screen identifies compounds with specific anti-wolbachial activity 
As no nematode cell lines have been established to date, tto rapidly identify compounds with anti-wolbachial activity we adapted and miniaturized an in vitro high-content imaging assay, which relied on Drosophila melanogaster cells naturally infected with $w \mathrm{Mel}$ strain of Wolbachia (25). In the adapted assay, we used the LDW1 cell line (26) and two fluorescence in situ hybridization (FISH) probes specific to Wolbachia 16S rRNA to unambiguously stain Wolbachia and measure bacterial load inside host cells (Fig. 1A-C). Wolbachia are sensitive to tetracycline and rifamycin antibiotics $(27,28)$ and these controls demonstrated specific antiwolbachial activity in the assay, with doxycycline's half maximal inhibitory concentration $\mathrm{IC}_{50}=$ $279 \mathrm{nM}$ and rifampicin $\mathrm{IC}_{50}=5 \mathrm{nM}$ (fig. 1D). Optimized assay conditions yielded a robust assay with Z' factors > 0.5 (table S1). Further miniaturization to 1536-well format did not reduce assay quality (fig. 1C and table 1). Using this optimized assay, we screened 300,368 small molecules from established libraries, including ReFRAME (29), for anti-wolbachial activity (table S1), with an overall hit rate of $0.70 \%$. Reconfirmed hits were tested against Wolbachia in dose response and for cytotoxicity in the mammalian HEK293T and HepG2 cell lines (table S1). Overall, we identified and reconfirmed 299 potent $\left(\mathrm{IC}_{50}<1 \mu \mathrm{M}\right.$ ) and selective (half maximal cytotoxic concentration $\left.\left(\mathrm{CC}_{50}\right): \mathrm{IC}_{50}>10\right)$ anti-wolbachial compounds (fig. 1D-G, H, table S1).

We identified a number of known drugs and bioactive molecules among our potent and selective hits including antibiotics, signal transduction modulators, antineoplastics, antifungals, and antivirals (fig. 2, fig. S1, table S2).Antibiotics made up the largest category (46\%) of the identified known drugs, and included tetracyclines, rifamycins, peptide deformylase inhibitors, pleuromutilins, fluoroquinolones, and aminocoumarins. Many of these displayed exquisite potencies against Wolbachia in vitro (e.g., coumermycin $\left.\mathrm{IC}_{50}=1.5 \mathrm{nM}\right)$. Among the antiwolbachial antibiotic hits we also identified macrolide antibiotics tylosin and its derivative tylvalosin, with $w \mathrm{Mel} \mathrm{IC}_{50} \mathrm{~s}$ of $720 \mathrm{nM}$ and $350 \mathrm{nM}$, respectively. However, poor bioavailability, previously identified toxicity liabilities, challenging and costly synthesis, and most importantly lack of retained activity against filarial Wolbachia made these unattractive for repurposing or further development.

We also identified a number of novel compounds among the hits. To determine if these anti-wolbachial hits were Wolbachia-specific and/or possessed antibiotic activity we screened them against a panel of extracellular bacteria. As expected, antibiotics and known drugs showed 
activity against many bacterial species, but very few novel small molecules inhibited microbial growth, even at the highest concentration tested of $20 \mu \mathrm{M}$ (fig. 2, table S3). This suggests that the novel chemical scaffolds identified in our screen had Wolbachia-specific activity or acted on a host cell process required for Wolbachia's intracellular survival. Likewise, the optimized quinazoline leads CBR417 and CBR490 did not generally inhibit extracellular bacterial growth (table S4).

A worm-based ex vivo assay rapidly identifies hits with anti-filarial Wolbachia activity

Our primary cell-based assay identified compounds with activity against $w \mathrm{Mel}$, a strain of Wolbachia that infects D. melanogaster. Validation of anti-wolbachial compound activity against filarial Wolbachia commonly requires in vivo models, which is not amenable to rapid compound optimization cycles and has impeded drug discovery efforts. To overcome this limitation, we developed an orthogonal ex vivo validation assay that would allow us to prioritize hits in a native context against filarial Wolbachia (fig. 3, fig. 4A, fig. S2,). Based on previous studies (25), we selected quantification of filarial Wolbachia stained via 16S rRNA FISH near a convenient landmark for quantification, the ovary distal tip cell (DTC), of B. pahangi to validate our primary screen hits. The Wolbachia distribution in the ovaries is predictable and consistent compared to the variable distribution in hypodermal chords, with highest concentrations near the DTC (fig. 3A-F, fig. S2A, B, (30)), This population appears more refractive to compound treatment in the three day ex vivo assay compared to the population found in the hypodermis (fig. 3G, H), as has also been observed in Onchocerca ochengi worms in vivo (31). Moreover, the reproductive tract is a relevant site for anti-wolbachial drug action, and clearance in germ line stem cells is likely critical to prevent recrudescence of the bacteria following cessation of treatment.

Doxycycline treatment of up to $9 \mu \mathrm{M}$ was insufficient to completely clear $w \mathrm{Bp}$ from $B$. pahangi ovaries in three days, but $1 \mu \mathrm{M}$ and $3 \mu \mathrm{M}$ treatments eliminated approximately $75 \%$ of Wolbachia, with an estimated half maximal effective concentration $\left(\mathrm{EC}_{50}\right)$ of $441 \pm 64 \mathrm{nM}$ (fig. 4B-D). A similar result was observed with $w \mathrm{Bm}$ strain of Wolbachia in B. malayi nematodes, where a $1 \mu \mathrm{M}$ doxycycline treatment eliminated $72.5 \%$ of Wolbachia (fig. S3). Benchmarking on this doxycycline activity, we established a validation threshold for our candidate anti-wolbachial 
compounds of $\geq 75 \% \mathrm{wBp}$ elimination from the distal ovary at a compound concentration of 1 $\mu \mathrm{M}$ (fig. 4B).

The ex vivo validation assay could be performed in eleven days, considerably reducing optimization cycle times versus the three month long in vivo validation assays, but was labor intensive, relying on nematode dissection and confocal imaging. Thus, based on activity and structural similarity clustering, we chose to test 137 out of our 299 primary hits (i.e., the most potent of any closely related analogs) (fig. $4 \mathrm{E}$ ). Of these, 32 showed $\mathrm{a} \geq 75 \% \mathrm{wBp}$ elimination at $1 \mu \mathrm{M}$, for a validation rate of $23.4 \%$ (table S1, table S5). The percent elimination of $w \mathrm{Bp}$ in worm ovaries was not generally correlated to compound potency observed in the primary $D$. melanogaster cell-based assay $\left(\mathrm{R}^{2}=0.00048\right)$ (fig. 4F). Motility of worms was not affected by majority of compounds assayed, with the exception of methylene blue (table S2). Structure analysis of novel molecules demonstrated an enrichment of a quinazoline scaffold among validated compounds: out of eleven quinazolines tested in the secondary assay, eight quinazolines showed activity superior to doxycycline (fig. 4F). Members of this series displayed in vitro activity that correlated more with their activity in the ex vivo validation assay $\left(\mathrm{R}^{2}=\right.$ $0.3658)$ compared to all validated compounds $\left(\mathrm{R}^{2}=0.0023\right)$ (fig. $\left.4 \mathrm{G}\right)$. As quinazoline heterocycles are present in many of biologically active compounds including antibacterials (32, 33), we focused on this series to improve their physiochemical properties and metabolic stability, and their activity against filarial Wolbachia.

Quinazoline series demonstrates potent anti-filarial Wolbachia activity ex vivo and drug-like properties

We carried out a medicinal chemistry campaign to optimize the potency, safety, and physiochemical and pharmacokinetic properties of the quinazoline series, starting with the screen hit CBR008. This involved iterative profiling of analogues in the in vitro cell-based and ex vivo worm-based assays to determine their anti-wolbachial activity. Compounds with $\geq 90 \% w \mathrm{Bp}$ elimination at $1 \mu \mathrm{M}$ in the worm-based assay underwent absorption, distribution, metabolism, and excretion (ADME) profiling to assess cytochrome P450 (CYP) and human ether-a-go-go related gene (hERG) inhibition (to understand potential drug-drug interaction and cardiotoxicity liabilities of the compounds, respectively), metabolism in human and mouse liver microsomes, 
permeability in Caco-2 cells, kinetic solubility and plasma protein binding. Analogues with favorable properties (CBR422, CBR625, CBR715, CBR417, CBR490) were advanced for pharmacokinetic studies in mice to determine if their profiles were suitable for once-a-day (QD) or twice-a-day (BID) dosing in the in vivo preclinical models of infection (e.g. when dosed orally in mice at $\leq 50 \mathrm{mg} / \mathrm{kg}$ maintained plasma exposure over their $w \mathrm{Bp} \mathrm{EC}_{90}$ s for at least 8 hours (h)). As for doxycycline, the quinazoline series compounds had comparable antiwolbachial activity in B. pahangi and B. malayi worms in the ex vivo assay (determined for CBR422 and CBR625; fig. S3).

Briefly, we found that replacing the amide with an oxadiazole isostere or methylpyridine at the $\mathrm{C} 2$ position of the quinazoline core and the trifluoromethyl with a pentafluorosulfanyl group improved the in vitro and ex vivo potencies, while increasing metabolic stability and pharmacokinetic properties of the compounds. This effort led to the initial lead CBR422, and the advanced (CBR625, CBR715) and optimized (CBR417, CBR490) quinazolines that had excellent potency, selectivity and ADME properties (table 1, table S6). Specifically, compared to screen hit CBR008 these analogues demonstrated improved in vitro and ex vivo potencies ( $w \mathrm{Mel}$ $\mathrm{IC}_{50} \leq 33 \mathrm{nM} ; w \mathrm{Bp} \mathrm{EC}_{50} \leq 356 \mathrm{nM}$ ), an acceptable selectivity index, were orally bioavailable in mice, and had excellent pharmacokinetic properties with a prolonged blood plasma exposure time over $\mathrm{EC}_{90}$ when dosed at $\leq 50 \mathrm{mg} / \mathrm{kg}$ (>12 h to $>24 \mathrm{~h}$ ) (table S7). The identified CYP and hERG liabilities of the series (CYP 1A2 IC 50 inhibition of $0.33 \mu \mathrm{M}$ for initial lead CBR422 and hERG inhibition $\mathrm{IC}_{50}$ of $5 \mu \mathrm{M}$ for CBR625) were markedly reduced in CBR417 (CYP inhibition $\geq 30 \mu \mathrm{M}$ for all isoforms, $\mathrm{hERG} \mathrm{IC}_{50}=19.5 \mu \mathrm{M}$ ) and partially addressed in CBR490 (CYP 1A2 inhibition $\mathrm{IC}_{50}=6.4 \mu \mathrm{M}$, hERG inhibition $\mathrm{IC}_{50}$ of $7 \mu \mathrm{M}$ ), while low kinetic solubility and high protein binding continued to be a feature of the analogs. Importantly, the advanced and optimized quinazolines were selective against $L$. loa microfilariae (that do not contain Wolbachia) in an in vitro motility assay, with $\mathrm{IC}_{50} \mathrm{~s}$ of $>100 \mu \mathrm{M}$ for $\mathrm{CBR} 715,87 \mu \mathrm{M}$ for CBR417, and $64 \mu \mathrm{M}$ for CBR490 versus the $11.3 \mu \mathrm{M}$ IC $_{50}$ of ivermectin (table 1).

Optimized quinazoline series demonstrates in vivo efficacy with shortened duration of treatment in preclinical model of filarial infection 
A gold-standard in vivo preclinical model for assessing activity of anti-filarial compounds within a reasonable period of time utilizes mice infected with a filarial parasite of rodents, Litomosoides sigmodontis (fig. 6A) (16, 34). As L. sigmodontis are hosts to the Wolbachia endosymbiont, this is also an excellent preclinical model to assess anti-wolbachial compound action, which is done using quantitative PCR (qPCR) to determine the Wolbachia ftsZ gene to the L. sigmodontis actin gene ratio in female adult worms recovered from mice at the end of the experiment (4-6 weeks post treatment start; 65-77 days post infection). A series of studies in this model demonstrated that quinazoline potency in the ex vivo worm-based assay together with the ability of the compounds to achieve good exposure following oral dosing were essential for achieving efficacy. For example, an early analog CBR063 with good ex vivo potency $\left(\mathrm{IC}_{50}=\right.$ $89 \mathrm{nM} ; \mathrm{EC}_{50}=97 \mathrm{nM}$ ) failed to achieve Wolbachia clearance in vivo (fig. 6B), likely due to a comparably inferior pharmacokinetic profile $\left(\mathrm{C}_{\max }=119 \pm 38.3 \mathrm{ng} / \mathrm{mL}\right)$ (table $\left.\mathrm{S} 7\right)$. However, quinazoline analogues CBR422, CBR625, CBR715, CBR417, and CBR490 with excellent ex vivo potency and pharmacokinetic profiles, all proved efficacious in vivo with $<14$ day $\leq 60$ $\mathrm{mg} / \mathrm{kg}$ BID dosing regimens (> 99\% median Wolbachia clearance) and were significantly (Pvalue $<0.05$ to $<0.0001$ ) superior to the 14 -day $40 \mathrm{mg} / \mathrm{kg}$ BID doxycycline control ran in parallel (fig. 6B, fig. S5, table 2).

Ability to achieve efficacy in preclinical models with a shortened duration of treatment $(\leq$ 7 days) is a desired profile because a reduced dosing schedule for an anti-wolbachial medication has the potential to facilitate treatment and improve compliance. Therefore, we explored shortened treatment regimens for the efficacious quinazoline analogs. Efficacy $(99.80 \%$ Wolbachia elimination) was achieved with 60 mg/kg CBR625 BID 7-day dosing, and near-target efficacy (98.95\% elimination) with $60 \mathrm{mg} / \mathrm{kg}$ QD dosing (fig. S5A, table 2). Likewise, an oral 7and 12-day treatment of CBR715 at $50 \mathrm{mg} / \mathrm{kg}$ BID eliminated $98.86 \%$ and $99.80 \%$ of Wolbachia, respectively (fig. 6B, table 2). Sparse PK sampling during in vivo studies confirmed relative exposures of the tested quinazolines (fig. S4). Furthermore, an oral 4-day treatment at 60 $\mathrm{mg} / \mathrm{kg}$ QD with the optimized quinazolines eliminated 99.80\% (CBR490) and 99.96\% (CBR417) of Wolbachia in L. sigmodontis adult female worms, significantly superior ( $\mathrm{P}$-value $=0.0013$ for CBR490 and P-value < 0.0001 for CBR417) to the 14-day doxycycline control ran in parallel (95.21\% elimination) (fig. 6B, table 2). 
Due to the demonstrated potency and favorable exposures of optimized quinazolines CBR417 and CBR490 (time over EC90 of $72 \mathrm{~h}$ for a single $100 \mathrm{mg} / \mathrm{kg}$ PO dose; table S7)), we investigated whether an even more drastically abbreviated efficacious dosing regimen with these compounds were attainable. Both CBR417 and CBR490 were dosed at $100 \mathrm{mg} / \mathrm{kg}$ once per week over a two-week period ( 2 doses total) in the mouse/L. sigmodontis model and a $200 \mathrm{mg} / \mathrm{kg}$ single-dose was also evaluated. All treatment regimens eliminated $>99 \%$ of Wolbachia in L. sigmodontis adult female worms, significantly superior $(\mathrm{P}$-value $<0.01)$ to the 14 -day doxycycline control ran in parallel (95.21\% elimination) (fig. 6B, table 2). Examination of in vivo efficacy in response to diverse dosing regimens showed that the dose of CBR490 was equally correlated to in vivo efficacy $\left(\mathrm{R}^{2}=0.7263\right)$ as was total dose $\left(\mathrm{R}^{2}=0.713\right)$ (fig. S6). Too few data points were available for CBR417 to make a conclusive analysis.

\section{CBR417 and CBR490 demonstrate favorable safety profiles in preclinical studies}

CBR417 and CBR490 safety profiles were more extensively assessed (table 3). Both compounds were well tolerated in mouse in vivo efficacy studies, even when administered at high doses $200 \mathrm{mg} / \mathrm{kg}$ (single dose) or for prolonged periods of time (CBR490 $60 \mathrm{mg} / \mathrm{kg}$ daily total dose for 11 days). Neither compound showed intrinsic mutagenic potential based on negative results in mini-Ames, either in the absence or presence of rat liver S9 mix for metabolic activation. Micronucleus assays also did not reveal inherent genotoxicity potential. The CBR417 oxadiazole did not strongly inhibit hERG or CYP enzymes (the latter assessed for potential drugdrug interactions) and neither compound caused human PXR activation (a hallmark of CYP3A4 induction). A prospective cardiovascular liability due to hERG inhibition was identified for CBR490 in preliminary profiling assays $\left(\mathrm{IC}_{50}=7.07 \mu \mathrm{M}\right)$; however, a cardiac safety panel revealed no significant hits for either compound (table S8). To explore other potential off-target effects that could lead to in vivo toxicity, the Eurofins Cerep-Panlabs safety screen against 44 selected targets was carried out and identified twelve targets significantly inhibited (> 50\%) by CBR490 and only three inhibited by CBR417 (table S9). In summary, these findings demonstrate the favorable safety profiles of CBR417 and CBR490 quinazolines. 
Advanced quinazoline lead eliminates Wolbachia in B. malayi and Onchocerca adult worms in vivo

Due to the demonstrated efficacy of optimized quinazoline analogues against Wolbachia in L. sigmodontis, we assayed one of these advanced leads (CBR715) for efficacy in preclinical murine models of B. malayi and Onchocerca adult worm infections. Retention of compound activity in the Onchocerca model was of particular concern, as both the Wolbachia endosymbionts and the Onchocerca hosts are more distantly related from the above host/endosymbiont models: Wolbachia of Onchocerca species belong to Supergroup C while Wolbachia of the more closely related L. sigmodontis and Brugia spp. belong to Supergroup D (35-37).The only available in vivo model of Onchocerca adult worms utilizes the bovine parasite O. ochengi (38), which is a sister species and the closest relative of the human river blindness parasite, O. volvulus (39).

The SCID mouse B. malayi and $O$. ochengi models were previously described, and like the mouse/L. sigmodontis efficacy model, rely on qPCR for quantification of filarial Wolbachia (38). In the B. malayi in vivo model (fig. 7A), treatments with CBR715 (7- and 14-day dosing schedules at $50 \mathrm{mg} / \mathrm{kg}$ BID) eliminated $>99 \%$ Wolbachia in B. malayi adult females, with the 14-day CBR715 treatment eliminating significantly more Wolbachia $(\mathrm{P}$-value $=0.0464)$ compared to the 42-day doxycycline control (fig. 7B). Doxycycline (42-day) and both CBR715 treatments eliminated all circulating microfilariae (fig. 7C) and although a general trend of reduced adult worm burden was observed, these differences were not statistically significant (Pvalue > 0.05) (fig. 7D). Likewise, in the $O$. ochengi in vivo model (fig. 7E), 7- and 14-day treatments with CBR715 eliminated $>99 \%$ Wolbachia in O. ochengi adult males, on par with the 28-day doxycycline treatment control (fig. 7F), and no difference in percent recovery of implanted males was observed (fig. 7G). These data confirm the broad spectrum of activity of the optimized anti-wolbachial quinazoline series, and the continued superior performance of this series compared to doxycycline in in vivo preclinical rodent models of diverse filarial infections.

\section{Discussion}


Here we describe an accelerated drug discovery platform for the identification of antiwolbachial compounds and translation of these to efficacious leads in in vivo models of filarial infection. Previous screening efforts using high-throughput insect cell-based assays have identified anti-wolbachial compounds active in vitro, yet translation of these hits to in vivo models and the clinic has been challenging for a number of reasons. First, Wolbachia are obligate intracellular bacteria, and may only be propagated within appropriate host cells. As no nematode cell lines have been developed, for high throughput screening drug discovery researchers have relied on insect cell lines infected with Wolbachia strains that are specific to these hosts $(25,28,40-42)$. Fortuitously, there are substantial similarities in the genetics and cell biology of the Wolbachia species that warrant using the infected insect cell lines as a primary screen and a high-throughput proxy for filarial Wolbachia-based assays. However, there are also considerable differences between Wolbachia strains, demonstrated not only by host range, but also by their genomes. For example, Foster et al reported a greater reduction of B. malayi Wolbachia $w \mathrm{Bm}$ genome (in total size and predicted gene number) compared to D. melanogaster Wolbachia w Mel (43). Thus, compounds identified in whole cell screens against insect Wolbachia may hit targets that are sufficiently divergent or even absent in filarial Wolbachia. Similarly, compounds that target a host cell factor to reduce Wolbachia load may be absent in filarial nematodes. Finally, filarial nematodes may shelter Wolbachia from compound action through limited permeability, compound metabolism, and/or excretion.

To address these limitations, following up on initial studies (25) we developed an orthogonal assay in filarial nematodes that allowed us to rapidly assess anti-filarial Wolbachia activity of our primary screen hits. We utilized B. pahangi, a filarial parasite of cats but which can also infect humans (44), because these worms are closely related to B. malayi (45), but can be maintained in an animal host (jirds) in larger quantities, and therefore are more readily available. We focused our evaluation of Wolbachia load within worm ovaries for a number of reasons, including because this population appeared less sensitive to compound treatment than Wolbachia in the hypodermal chords of the animals, providing a more rigorous and pertinent read out of anti-wolbachial compound action. Interestingly, a similar differential susceptibility to compound action in the hypodermis versus the ovaries has been previously observed in vivo in O. ochengi adult worms following antibiotic treatments in cattle (31). The difficulty in eliminating different clades of Wolbachia from ovaries of different species of worms (Wolbachia 
supergroup D in Brugia spp. reported here and supergroup C in Onchocerca spp.) further supports the relevance of this tissue for assessment of anti-wolbachial compound efficacy. We utilized Wolbachia 16S rRNA FISH to detect the bacteria in both the cell-based high throughput assay and the ex vivo worm-based assay. In addition to its inherent specificity, rRNA provides a more sensitive viability metric, as it is less stable than DNA, allowing us to observe Wolbachia elimination in worm ovaries after only a relatively short three-day treatment ex vivo. While true markers of viability can be challenging to utilize in high-throughput screens, this approach gave us confidence in our ability to select fast-acting, anti-filarial Wolbachia compounds.

In our primary insect cell-based screen, we identified known drugs with potent and selective anti-wolbachial activity. Among these were antineoplastics and signal transduction modulators, which potentially exert their activity by affecting host cell processes exploited or required by this obligate intracellular bacterium. For example Wolbachia has been found to alter lipid metabolism of mosquitoes (46), and insulin signaling and the TOR complex 1 pathway have been implicated in controlling Wolbachia titers in D. melanogaster $(47,48)$. Accordingly, in our screen we identified mTOR inhibitors and drugs affecting cellular metabolism (e.g., drugs for diabetes and liver $\mathrm{X}$ receptor agonists). We also identified many antibiotics with antiwolbachial activity, including ones belonging to antibiotic classes that have been previously identified in similar insect cell-based screens and assays (tetracyclines, rifamycins, pleuromutilins, fluoroquinolones, and macrolides (ABBV-4083, an orally available derivative of the macrolide antibiotic tylosin, is currently being developed as an anti-wolbachial therapy)) (27, $28,41,49,50)$, and others that to our knowledge have not been previously reported (aminocoumarins and peptide deformylase inhibitors). Notably, we found that most of these known drugs and antibiotics did not efficiently eliminate Wolbachia from B. pahangi ovaries in our ex vivo validation assay, regardless of their impressive potency in vitro. Therefore, while we relied on the high-throughput assay to identify potential anti-wolbachials, developing and utilizing an orthogonal assay that evaluated compound efficacy against Wolbachia in parasitic worms allowed us to prioritize molecules with rapid anti-filarial Wolbachia activity for further medicinal chemistry optimization.

The desired profile for an anti-wolbachial macrofilaricide compound is the ability to cause $>99 \%$ depletion of Wolbachia in adult worms within 7 days of dosing in all three preclinical models of filarial disease (L. sigmodontis, B. malayi and O. ochengi). The oxadiazole 
and methylpyridine leads (CBR625, CBR417 and CBR715, CBR490) proved efficacious in vivo, causing a $>99 \%$ Wolbachia elimination in adult L. sigmodontis worms within the mandated dosing schedule of $\leq 7$ days. Additionally, the advanced methylpyridine lead CBR715 recapitulated this in vivo efficacy against Wolbachia in human parasite $B$. malayi and a close surrogate of $O$. volvulus $(O$. ochengi), demonstrating real promise in translation of the quinazoline series to a cure for human filarial infections. Our optimization strategy ultimately led to synthesis of leads CBR490 and CBR417 that with just a single-dose were efficacious in vivo at eliminating $>99 \%$ of Wolbachia from adult L. sigmodontis female worms. Abbreviated dosing schedules have a real advantage in treating infections in resource limited countries and elsewhere, as compliance and point of care distribution is greatly facilitated. Finally, both CBR417 and CBR490 demonstrated safety in initial in vivo and in vitro preclinical profiling and did not show strong activity in vitro against $L$. loa microfilariae $\left(\mathrm{IC}_{50}=87 \mu \mathrm{M}\right.$ and $64 \mu \mathrm{M}$, respectively) compared to the ivermectin control $\left(\mathrm{IC}_{50}=11.3 \mu \mathrm{M}\right)$, suggesting that they would be appropriate for administration to patients in L. loa endemic regions following further safety assessments against L. loa microfilariae in vivo.

Despite the promise of these results, we note limitations and outstanding questions that need to be addressed before clinical translation of this work. It should be noted that due to the length of time (years) needed for adult worm death following Wolbachia elimination, reduction in worm numbers in the murine assays is not anticipated and was not observed. However, the more immediate phenotype of worm sterilization was observed in the B. malayi murine model. Currently we also have no evidence that the observed Wolbachia elimination is sustained beyond four to five weeks following treatment, and studies using in vivo jird models that can accommodate patent filarial infections for at least six months (51) are necessary to determine the lowest efficacious dose of quinazolines that prevent Wolbachia recrudescence. Lastly, treatment of the large, long-lived female worms belonging to the Onchocerca spp. represents the ultimate challenge, with females containing 20x more Wolbachia than males (52). Therefore, further assessment of quinazoline efficacy in models that support Onchocerca female worms in vivo (such as the bovine model of infection) will likewise be required to determine efficacious dosing regimens. 
Recently, chemical optimization of the thienopyrimidine series identified in highthroughput screening led to the generation of AWZ1066, a compound with a quinazoline scaffold and increased efficacy against both insect and filarial Wolbachia (53). However, quinazoline heterocycles are present in many biologically active compounds and whether CBR417, CBR490 and AWZ1066 share the same mechanism of action is uncertain. Genetic manipulation of Wolbachia has not been developed and its obligate intracellular lifestyle complicates target identification efforts, such as evolution of resistance and confirmation of putative targets via genetic means. Interestingly, AWZ1066 and many novel scaffolds identified in our primary screen, including the prototypical quinazoline CBR008, demonstrated very specific anti-bacterial spectrum of activity, which may indicate a Wolbachia- or Wolbachia hostspecific target. This also suggests that the quinazolines may be narrow spectrum antibiotics, a favorable profile for treating filarial nematode infections while reducing the effects of treatment on the microbiomes of treated individuals.

In summary, our anti-wolbachial drug development platform enabled the path towards a short course oral therapy for elimination of Wolbachia-reliant filarial nematodes, including ones that cause lymphatic filariasis and onchocerciasis. Our work supports advancement of the oxadiazole and methylpyridine quinazoline subseries for additional preclinical safety assessment and indicates that quinazolines are a selective treatment for currently intractable filarial worm infections.

\section{Materials and Methods}

\section{Study Design}

The objective of this study was to identify anti-filarial Wolbachia compounds with efficacy superior to that of doxycycline when administered with an abbreviated dosing schedule $(\leq 7$ days). Wolbachia-infected Drosophila cell-based high-content imaging assay was used to screen for potent anti-wolbachial compounds, and putative hits were counter-screened in mammalian cells. Activity of potent and selective compounds was validated in an ex vivo whole worm assay observing filarial Wolbachia reduction in B. pahangi adult female ovaries, benchmarking on doxycycline activity. In vivo experiments were designed to compare Wolbachia reduction in $L$. sigmodontis, $O$. ochengi, or B. malayi adult worms between different treatment groups, a gold 
standard doxycycline and a vehicle control, in a randomized design with multiple arms and shared controls. The Wolbachia single gene ftsZ/worm actin ratios were compared to the vehicle and doxycycline treatment. Where applicable, sample size, selection, blinding schemes and replicates are provided in the figure legends and Materials and Methods. Primary data are reported in data file S1.

\section{Primary in vitro cell-based assay}

Wolbachia-infected LDW1 cells (26) were maintained in Shields and Sang M3 (SSM3) Insect Medium (Sigma) supplemented with 10\% heat-inactivated Fetal Bovine Serum (FBS) (qualified, One Shot Format, Gibco) at $25^{\circ} \mathrm{C}$, in flasks with unvented caps. Assay plates (Greiner, Part. No. 789071 and 789091) were prepared by coating with $0.5 \mathrm{mg} / \mathrm{mL}$ (384-well plates) or $1 \mathrm{mg} / \mathrm{mL}$ (1536-well plates) solution of Concanavalin-A lectin (MP BIOMEDICALS). Compounds were acoustically transferred into coated plates using the Echo 555 Liquid Handler (Labcyte Inc.). Cells were trypsinized (TrypLE Express, Gibco), scraped, and seeded at 12,000 cells/well (384well plates) or 4,000 cells/well (1536-well plates) in SSM3 medium supplemented with 2\% FBS. Plates were spun at $800 \mathrm{rpm}$ for $3 \mathrm{~min}$ and incubated at $25^{\circ} \mathrm{C}$. Six days after seeding cells were fixed with $4 \%$ paraformaldehyde (PFA) for at least $10 \mathrm{~min}$ and washed with phosphate buffered saline, $\mathrm{pH} 7,0.1 \%$ Tween 20 (PBS-T). FISH was used to stain Wolbachia and $3 \mu \mathrm{M}$ DAPI was used to stain DNA. The MultiFlo FX Multi-Mode Dispenser (Biotek) was used for Concanavalin-A coating, cell fixation and staining of 384-well plates and the "bottle valve" dispenser with an angled-head (Kalypsys Inc.) was used for processing of 1536-well plates. Plates were imaged using the CX5 Insight Cellomics high content imaging instrument with a 10× objective (Thermo Fisher Scientific). Each well was analyzed using Compartmental Analysis in HCS Studio (Thermo Fisher Scientific) for cell number and Wolbachia content (see Supplementary Materials and Methods).

\section{Orthogonal ex vivo Brugia validation assay}

Adult B. pahangi and B. malayi females cultivated in and extracted from peritoneal cavities of jirds (Meriones unguiculatus) were obtained mainly from TRS Laboratories. B. pahangi were also provided by Dr. Brenda T. Beerntsen (University of Missouri), and the NIH/NIAID Filariasis Research Reagent Resource Center for distribution by BEI Resources, NIAID, NIH: 
Adult Female Brugia pahangi (Live), NR-48903. Following shipment, worms were immediately separated into 24-well plates, one animal per well, and allowed to recover for two days in high glucose RPMI 1640 Medium (ATCC modification) (Gibco) supplemented with 10\% MEM (Gibco) and 10\% heat-inactivated HyClone FBS (GE Healthcare Life Sciences). Media was changed daily and compounds tested at indicated concentrations (0.1\% DMSO). Gross motility of worms was observed by eye during treatment and compared to DMSO controls. After three days of treatment animals were frozen at $-80{ }^{\circ} \mathrm{C}$, thawed, and fixed for 20 min with $3.2 \%$ PFA in PBS-T. Ovaries were dissected out, stained for Wolbachia using a modified FISH protocol, mounted on slides using Vectashield with DAPI mounting medium (Vector Laboratories Inc., H1200), and imaged using a confocal microscope (see Supplementary Materials and Methods). To reduce variability, worms originating from a single jird were used in each experiment. The experiments were carried out partially blinded, as with the exception of DMSO and doxycycline controls identity of tested compounds was masked during treatment, imaging, and analysis.

\section{Statistical analysis}

Percentage Wolbachia reduction in macrofilariae were normalized to median vehicle control values derived from the same experimental infection and screen. Where available, repeat experimental data were pooled following normalization. For analysis of Wolbachia depletion in

in vivo experiments, where majority of grouped data failed the D'Agostino \& Pearson normality test (P-value > 0.05), a non-parametric Kruskal-Wallis test with Dunn's correction for multiple comparisons was used to determine significance, and medians with $95 \%$ confidence intervals are shown. Comparisons between vehicle and all treatment groups and doxycycline and all treatment groups were preselected. All statistics were computed using GraphPad Prism v6.0h.

\section{Supplementary Materials}

Supplementary Materials and Methods

Fig. S1. Classes of known drugs and bioactive molecules identified as potent and selective antiwolbachial hits in the primary in vitro screen.

Fig. S2. Wolbachia distribution in ovaries and the hypodermis of DMSO treated worms. 
Fig. S3. Wolbachia elimination from B. malayi worm ovaries ex vivo.

Fig. S4. Sparse pharmacokinetic profiles of quinazoline anti-wolbachials during efficacy studies.

Fig. S5. Wolbachia elimination following quinazoline treatment in the mouse/L. sigmodontis in vivo model of filarial infection.

Fig. S6. CBR417 and CBR490 dose-response relationship based on Wolbachia elimination in mouse/L. sigmodontis efficacy studies.

Table S1. Primary and validation screen statistics.

Table S2. Anti-wolbachial activities of known drugs and bioactive molecules identified as potent and selective hits in the primary in vitro screen (powders and ReFRAME compounds).

Table S3. Antibacterial activities of primary screen hits from Bioactive, Diversity I and Diversity II libraries.

Table S4. Activity of optimized anti-wolbachial leads against a panel of gram-positive and gramnegative bacteria.

Table S5. Activities of screening hits validated ex vivo (all except ReFRAME compounds are powders).

Table S6. ADMET properties of quinazoline anti-wolbachials.

Table S7. Pharmacokinetic properties of anti-wolbachial quinazolines.

Table S8. Cardiac Panel Study Results for CBR417 and CBR490.

Table S9. Safety Pharmacology Profiling Study Results for CBR417 and CBR490.

Data file S1. Primary data.

\section{References and Notes:}

1. C. Global Burden of Disease Study, Global, regional, and national incidence, prevalence, and years lived with disability for 301 acute and chronic diseases and injuries in 188 countries, 1990-2013: a systematic analysis for the Global Burden of Disease Study 2013. Lancet 386, 743-800 (2015).

2. WHO Department of control of neglected tropical diseases, "Investing to overcome the global impact of neglected tropical diseases: third WHO report on neglected diseases 2015.," WHO report on neglected tropical diseases (2015). 
3. Global Health Estimates 2014 Summary Tables: YLD by Cause, Age and Sex, 2000-2012 (2014).

4. D. Gems, Longevity and ageing in parasitic and free-living nematodes. Biogerontology 1, $289-307$ (2000).

5. J. Lawrence, Y. K. Sodahlon, K. T. Ogoussan, A. D. Hopkins, Growth, Challenges, and Solutions over 25 Years of Mectizan and the Impact on Onchocerciasis Control. PLoS neglected tropical diseases 9, e0003507 (2015).

6. M. Y. Osei-Atweneboana, K. Awadzi, S. K. Attah, D. A. Boakye, J. O. Gyapong, R. K. Prichard, Phenotypic evidence of emerging ivermectin resistance in Onchocerca volvulus. PLoS neglected tropical diseases 5, e998 (2011).

7. C. N. Pulaski, J. B. Malone, C. Bourguinat, R. Prichard, T. Geary, D. Ward, T. R. Klei, T. Guidry, G. Smith, B. Delcambre, J. Bova, J. Pepping, J. Carmichael, R. Schenker, R. Pariaut, Establishment of macrocyclic lactone resistant Dirofilaria immitis isolates in experimentally infected laboratory dogs. Parasites \& vectors 7, 494 (2014).

8. R. M. Kaplan, A. N. Vidyashankar, An inconvenient truth: global worming and anthelmintic resistance. Veterinary parasitology 186, 70-78 (2012).

9. R. J. Kastner, C. M. Stone, P. Steinmann, M. Tanner, F. Tediosi, What Is Needed to Eradicate Lymphatic Filariasis? A Model-Based Assessment on the Impact of Scaling Up Mass Drug Administration Programs. PLoS neglected tropical diseases 9, e0004147 (2015).

10. K. K. Frempong, M. Walker, R. A. Cheke, E. J. Tetevi, E. T. Gyan, E. O. Owusu, M. D. Wilson, D. A. Boakye, M. J. Taylor, N. K. Biritwum, M. Osei-Atweneboana, M. G. Basanez, Does Increasing Treatment Frequency Address Suboptimal Responses to Ivermectin for the Control and Elimination of River Blindness? Clinical infectious diseases : an official publication of the Infectious Diseases Society of America 62, 1338-1347 (2016).

11. B. V. Babu, G. R. Babu, Coverage of, and compliance with, mass drug administration under the programme to eliminate lymphatic filariasis in India: a systematic review. Transactions of the Royal Society of Tropical Medicine and Hygiene 108, 538-549 (2014).

12. L. Senyonjo, J. Oye, D. Bakajika, B. Biholong, A. Tekle, D. Boakye, E. Schmidt, E. Elhassan, Factors Associated with Ivermectin Non-Compliance and Its Potential Role in Sustaining Onchocerca volvulus Transmission in the West Region of Cameroon. PLoS neglected tropical diseases 10, e0004905 (2016).

13. D. H. Molyneux, A. Hopkins, M. H. Bradley, L. A. Kelly-Hope, Multidimensional complexities of filariasis control in an era of large-scale mass drug administration programmes: a can of worms. Parasites \& vectors 7, 363 (2014).

14. R. Stouthamer, J. A. Breeuwer, G. D. Hurst, Wolbachia pipientis: microbial manipulator of arthropod reproduction. Annual review of microbiology 53, 71-102 (1999).

15. S. C. Bosshardt, J. W. McCall, S. U. Coleman, K. L. Jones, T. A. Petit, T. R. Klei, Prophylactic activity of tetracycline against Brugia pahangi infection in jirds (Meriones unguiculatus). J Parasitol 79, 775-777 (1993).

16. A. Hoerauf, K. Nissen-Pahle, C. Schmetz, K. Henkle-Duhrsen, M. L. Blaxter, D. W. Buttner, M. Y. Gallin, K. M. Al-Qaoud, R. Lucius, B. Fleischer, Tetracycline therapy targets intracellular bacteria in the filarial nematode Litomosoides sigmodontis and results in filarial infertility. J Clin Invest 103, 11-18 (1999).

17. C. Bandi, T. J. Anderson, C. Genchi, M. L. Blaxter, Phylogeny of Wolbachia in filarial nematodes. Proceedings. Biological sciences / The Royal Society 265, 2407-2413 (1998).

18. M. J. Taylor, Wolbachia bacteria of filarial nematodes in the pathogenesis of disease and as a target for control. Trans R Soc Trop Med Hyg 94, 596-598 (2000).

19. A. Hoerauf, Filariasis: new drugs and new opportunities for lymphatic filariasis and onchocerciasis. Current opinion in infectious diseases 21, 673-681 (2008).

20. A. Hoerauf, S. Specht, M. Buttner, K. Pfarr, S. Mand, R. Fimmers, Y. Marfo-Debrekyei, P. Konadu, A. Y. Debrah, C. Bandi, N. Brattig, A. Albers, J. Larbi, L. Batsa, M. J. Taylor, O. Adjei, D. W. Buttner, Wolbachia endobacteria depletion by doxycycline as antifilarial therapy has macrofilaricidal activity in onchocerciasis: a randomized placebo-controlled study. Medical microbiology and immunology 197, 335 (2008).

21. M. J. Taylor, W. H. Makunde, H. F. McGarry, J. D. Turner, S. Mand, A. Hoerauf, Macrofilaricidal activity after doxycycline treatment of Wuchereria bancrofti: a double-blind, randomised placebo-controlled trial. Lancet 365, 2116-2121 (2005).

22. M. Walker, S. Specht, T. S. Churcher, A. Hoerauf, M. J. Taylor, M. G. Basanez, Therapeutic efficacy and macrofilaricidal activity of doxycycline for the treatment of river blindness. Clin Infect Dis 60, 1199-1207 (2015). 
23. J. D. Turner, S. Mand, A. Y. Debrah, J. Muehlfeld, K. Pfarr, H. F. McGarry, O. Adjei, M. J. Taylor, A. Hoerauf, A randomized, double-blind clinical trial of a 3-week course of doxycycline plus albendazole and ivermectin for the treatment of Wuchereria bancrofti infection. Clin Infect Dis 42, 1081-1089 (2006).

24. H. F. Cross, M. Haarbrink, G. Egerton, M. Yazdanbakhsh, M. J. Taylor, Severe reactions to filarial chemotherapy and release of Wolbachia endosymbionts into blood. Lancet 358, 1873-1875 (2001).

25. L. R. Serbus, F. Landmann, W. M. Bray, P. M. White, J. Ruybal, R. S. Lokey, A. Debec, W. Sullivan, A cell-based screen reveals that the albendazole metabolite, albendazole sulfone, targets Wolbachia. PLoS pathogens 8, e1002922 (2012).

26. P. M. White, L. R. Serbus, A. Debec, A. Codina, W. Bray, A. Guichet, R. S. Lokey, W. Sullivan, Reliance of Wolbachia on High Rates of Host Proteolysis Revealed by a Genome-Wide RNAi Screen of Drosophila Cells. Genetics 205, 1473-1488 (2017).

27. F. Fenollar, M. Maurin, D. Raoult, Wolbachia pipientis growth kinetics and susceptibilities to 13 antibiotics determined by immunofluorescence staining and real-time PCR. Antimicrob Agents Chemother 47, 16651671 (2003).

28. P. G. Hermans, C. A. Hart, A. J. Trees, In vitro activity of antimicrobial agents against the endosymbiont Wolbachia pipientis. J Antimicrob Chemother 47, 659-663 (2001).

29. J. Janes, M. E. Young, E. Chen, N. H. Rogers, S. Burgstaller-Muehlbacher, L. D. Hughes, M. S. Love, M. V. Hull, K. L. Kuhen, A. K. Woods, S. B. Joseph, H. M. Petrassi, C. W. McNamara, M. S. Tremblay, A. I. Su, P. G. Schultz, A. K. Chatterjee, The ReFRAME library as a comprehensive drug repurposing library and its application to the treatment of cryptosporidiosis. Proceedings of the National Academy of Sciences, (2018).

30. V. Foray, M. M. Perez-Jimenez, N. Fattouh, F. Landmann, Wolbachia Control Stem Cell Behavior and Stimulate Germline Proliferation in Filarial Nematodes. Developmental cell 45, 198-211 e193 (2018).

31. G. S. Bah, E. L. Ward, A. Srivastava, A. J. Trees, V. N. Tanya, B. L. Makepeace, Efficacy of three-week oxytetracycline or rifampin monotherapy compared with a combination regimen against the filarial nematode Onchocerca ochengi. Antimicrob Agents Chemother 58, 801-810 (2014).

32. V. Alagarsamy, K. Chitra, G. Saravanan, V. R. Solomon, M. T. Sulthana, B. Narendhar, An overview of quinazolines: Pharmacological significance and recent developments. European journal of medicinal chemistry 151, 628-685 (2018).

33. I. Khan, S. Zaib, S. Batool, N. Abbas, Z. Ashraf, J. Iqbal, A. Saeed, Quinazolines and quinazolinones as ubiquitous structural fragments in medicinal chemistry: An update on the development of synthetic methods and pharmacological diversification. Bioorganic \& medicinal chemistry 24, 2361-2381 (2016).

34. S. Specht, K. M. Pfarr, S. Arriens, M. P. Hubner, U. Klarmann-Schulz, M. Koschel, S. Sternberg, C. Martin, L. Ford, M. J. Taylor, A. Hoerauf, Combinations of registered drugs reduce treatment times required to deplete Wolbachia in the Litomosoides sigmodontis mouse model. PLoS neglected tropical diseases 12, e0006116 (2018).

35. M. Gerth, M. T. Gansauge, A. Weigert, C. Bleidorn, Phylogenomic analyses uncover origin and spread of the Wolbachia pandemic. Nature communications 5, 5117 (2014).

36. F. Comandatore, R. Cordaux, C. Bandi, M. Blaxter, A. Darby, B. L. Makepeace, M. Montagna, D. Sassera, Supergroup C Wolbachia, mutualist symbionts of filarial nematodes, have a distinct genome structure. Open biology 5, 150099 (2015).

37. M. Blaxter, G. Koutsovoulos, The evolution of parasitism in Nematoda. Parasitology 142 Suppl 1, S26-39 (2015).

38. A. Halliday, A. F. Guimaraes, H. E. Tyrer, H. M. Metuge, C. N. Patrick, K. O. Arnaud, T. D. Kwenti, G. Forsbrook, A. Steven, D. Cook, P. Enyong, S. Wanji, M. J. Taylor, J. D. Turner, A murine macrofilaricide pre-clinical screening model for onchocerciasis and lymphatic filariasis. Parasit Vectors 7, 472 (2014).

39. R. Morales-Hojas, R. A. Cheke, R. J. Post, Molecular systematics of five Onchocerca species (Nematoda: Filarioidea) including the human parasite, O. volvulus, suggest sympatric speciation. Journal of helminthology 80, 281-290 (2006).

40. K. L. Johnston, D. A. N. Cook, N. G. Berry, W. David Hong, R. H. Clare, M. Goddard, L. Ford, G. L. Nixon, P. M. O'Neill, S. A. Ward, M. J. Taylor, Identification and prioritization of novel anti-Wolbachia chemotypes from screening a 10,000-compound diversity library. Science advances $\mathbf{3}$, eaao1551 (2017).

41. K. L. Johnston, L. Ford, I. Umareddy, S. Townson, S. Specht, K. Pfarr, A. Hoerauf, R. Altmeyer, M. J. Taylor, Repurposing of approved drugs from the human pharmacopoeia to target Wolbachia endosymbionts of onchocerciasis and lymphatic filariasis. Int J Parasitol Drugs Drug Resist 4, 278-286 (2014). 
42. R. H. Clare, D. A. Cook, K. L. Johnston, L. Ford, S. A. Ward, M. J. Taylor, Development and validation of a high-throughput anti-Wolbachia whole-cell screen: a route to macrofilaricidal drugs against onchocerciasis and lymphatic filariasis. Journal of biomolecular screening 20, 64-69 (2015).

43. J. Foster, M. Ganatra, I. Kamal, J. Ware, K. Makarova, N. Ivanova, A. Bhattacharyya, V. Kapatral, S. Kumar, J. Posfai, T. Vincze, J. Ingram, L. Moran, A. Lapidus, M. Omelchenko, N. Kyrpides, E. Ghedin, S. Wang, E. Goltsman, V. Joukov, O. Ostrovskaya, K. Tsukerman, M. Mazur, D. Comb, E. Koonin, B. Slatko, The Wolbachia genome of Brugia malayi: endosymbiont evolution within a human pathogenic nematode. PLoS biology 3, e121 (2005).

44. A. Muslim, M. Y. Fong, R. Mahmud, S. Sivanandam, Vector and reservoir host of a case of human Brugia pahangi infection in Selangor, peninsular Malaysia. Tropical biomedicine 30, 727-730 (2013).

45. Y. L. Lau, W. C. Lee, J. Xia, G. Zhang, R. Razali, A. Anwar, M. Y. Fong, Draft genome of Brugia pahangi: high similarity between B. pahangi and B. malayi. Parasites \& vectors 8, 451 (2015).

46. J. C. Molloy, U. Sommer, M. R. Viant, S. P. Sinkins, Wolbachia Modulates Lipid Metabolism in Aedes albopictus Mosquito Cells. Applied and environmental microbiology 82, 3109-3120 (2016).

47. L. R. Serbus, P. M. White, J. P. Silva, A. Rabe, L. Teixeira, R. Albertson, W. Sullivan, The impact of host diet on Wolbachia titer in Drosophila. PLoS pathogens 11, e1004777 (2015).

48. T. Ikeya, S. Broughton, N. Alic, R. Grandison, L. Partridge, The endosymbiont Wolbachia increases insulin/IGF-like signalling in Drosophila. Proceedings. Biological sciences / The Royal Society 276, 37993807 (2009).

49. R. T. Jacobs, Lunde, C., Freund, Y.R., Hernandez, V., Li, X., Xia, Y., Berry, P.W., Halladay, J., Stefanakis, R., Easom, E.E., Plattner, J.J., Ford, L., Johnston, K.L., Cook, D.A.N., Clare, R., Cassidy, A., Myhill, L., Tyrer, H., Gamble, G., Guimaraes, A. F., Steven, A., Lenz, F., Ehrens, A., Frohberger, S.J., Koschel, M., Hoerauf, A., Hübner, M.P, McNamara, C., Bakowski, M.A., Turner, J.D., Taylor, M.J., Ward, S.A., Boron-Pleuromutilins as Anti-Wolbachia Agents with Potential for Treatment of Onchocerciasis and Lymphatic Filariasis. Journal of Medicinal Chemistry, In Press (2018).

50. M. J. Taylor, T. W. von Geldern, L. Ford, M. P. Hubner, K. Marsh, K. L. Johnston, H. T. Sjoberg, S. Specht, N. Pionnier, H. E. Tyrer, R. H. Clare, D. A. N. Cook, E. Murphy, A. Steven, J. Archer, D. Bloemker, F. Lenz, M. Koschel, A. Ehrens, H. M. Metuge, V. C. Chunda, P. W. Ndongmo Chounna, A. J. Njouendou, F. F. Fombad, R. Carr, H. E. Morton, G. Aljayyoussi, A. Hoerauf, S. Wanji, D. J. Kempf, J. D. Turner, S. A. Ward, Preclinical development of an oral anti-Wolbachia macrolide drug for the treatment of lymphatic filariasis and onchocerciasis. Sci Transl Med 11, (2019).

51. C. P. Morris, H. Evans, S. E. Larsen, E. Mitre, A comprehensive, model-based review of vaccine and repeat infection trials for filariasis. Clin Microbiol Rev 26, 381-421 (2013).

52. J. Gilbert, C. K. Nfon, B. L. Makepeace, L. M. Njongmeta, I. M. Hastings, K. M. Pfarr, A. Renz, V. N. Tanya, A. J. Trees, Antibiotic chemotherapy of onchocerciasis: in a bovine model, killing of adult parasites requires a sustained depletion of endosymbiotic bacteria (Wolbachia species). The Journal of infectious diseases 192, 1483-1493 (2005).

53. W. D. Hong, F. Benayoud, G. L. Nixon, L. Ford, K. L. Johnston, R. H. Clare, A. Cassidy, D. A. N. Cook, A. Siu, M. Shiotani, P. J. H. Webborn, S. Kavanagh, G. Aljayyoussi, E. Murphy, A. Steven, J. Archer, D. Struever, S. J. Frohberger, A. Ehrens, M. P. Hubner, A. Hoerauf, A. P. Roberts, A. T. M. Hubbard, E. W. Tate, R. A. Serwa, S. C. Leung, L. Qie, N. G. Berry, F. Gusovsky, J. Hemingway, J. D. Turner, M. J. Taylor, S. A. Ward, P. M. O'Neill, AWZ1066S, a highly specific anti-Wolbachia drug candidate for a short-course treatment of filariasis. Proceedings of the National Academy of Sciences of the United States of America 116, 1414-1419 (2019).

54. A. Heddi, A. M. Grenier, C. Khatchadourian, H. Charles, P. Nardon, Four intracellular genomes direct weevil biology: nuclear, mitochondrial, principal endosymbiont, and Wolbachia. Proceedings of the National Academy of Sciences of the United States of America 96, 6814-6819 (1999).

55. B. M. Fuchs, G. Wallner, W. Beisker, I. Schwippl, W. Ludwig, R. Amann, Flow cytometric analysis of the in situ accessibility of Escherichia coli 16S rRNA for fluorescently labeled oligonucleotide probes. Applied and environmental microbiology 64, 4973-4982 (1998).

56. M. A. Perotti, H. K. Clarke, B. D. Turner, H. R. Braig, Rickettsia as obligate and mycetomic bacteria. FASEB journal : official publication of the Federation of American Societies for Experimental Biology 20, 2372-2374 (2006).

57. T. Koressaar, M. Remm, Enhancements and modifications of primer design program Primer3. Bioinformatics 23, 1289-1291 (2007). 
58. A. Untergasser, I. Cutcutache, T. Koressaar, J. Ye, B. C. Faircloth, M. Remm, S. G. Rozen, Primer3--new capabilities and interfaces. Nucleic acids research 40, e115 (2012).

59. B. W. Li, Z. Wang, A. C. Rush, M. Mitreva, G. J. Weil, Transcription profiling reveals stage- and functiondependent expression patterns in the filarial nematode Brugia malayi. BMC genomics 13, 184 (2012).

60. M. W. Pfaffl, A new mathematical model for relative quantification in real-time RT-PCR. Nucleic acids research 29, e45 (2001).

61. L. Volkmann, O. Bain, M. Saeftel, S. Specht, K. Fischer, F. Brombacher, K. I. Matthaei, A. Hoerauf, Murine filariasis: interleukin 4 and interleukin 5 lead to containment of different worm developmental stages. Medical microbiology and immunology 192, 23-31 (2003).

62. J. D. Turner, R. Sharma, G. Al Jayoussi, H. E. Tyrer, J. Gamble, L. Hayward, R. S. Priestley, E. A. Murphy, J. Davies, D. Waterhouse, D. A. N. Cook, R. H. Clare, A. Cassidy, A. Steven, K. L. Johnston, J. McCall, L. Ford, J. Hemingway, S. A. Ward, M. J. Taylor, Albendazole and antibiotics synergize to deliver short-course anti-Wolbachia curative treatments in preclinical models of filariasis. Proceedings of the National Academy of Sciences of the United States of America 114, E9712-E9721 (2017).

63. D. Zofou, F. F. Fombad, N. V. T. Gandjui, A. J. Njouendou, A. J. Kengne-Ouafo, P. W. Chounna Ndongmo, F. R. Datchoua-Poutcheu, P. A. Enyong, D. T. Bita, M. J. Taylor, J. D. Turner, S. Wanji, Evaluation of in vitro culture systems for the maintenance of microfilariae and infective larvae of Loa loa. Parasites \& vectors 11, 275 (2018).

64. S. Wanji, E. E. Eyong, N. Tendongfor, C. Ngwa, E. Esuka, A. Kengne-Ouafo, F. Datchoua-Poutcheu, P. Enyong, A. Hopkins, C. D. Mackenzie, Parasitological, Hematological and Biochemical Characteristics of a Model of Hyper-microfilariaemic Loiasis (Loa loa) in the Baboon (Papio anubis). PLoS neglected tropical diseases 9, e0004202 (2015).

65. O. A. Fahmi, J. L. Raucy, E. Ponce, S. Hassanali, J. M. Lasker, Utility of DPX2 cells for predicting CYP3A induction-mediated drug-drug interactions and associated structure-activity relationships. Drug metabolism and disposition: the biological fate of chemicals 40, 2204-2211 (2012).

66. J. Bowes, A. J. Brown, J. Hamon, W. Jarolimek, A. Sridhar, G. Waldron, S. Whitebread, Reducing safetyrelated drug attrition: the use of in vitro pharmacological profiling. Nature reviews. Drug discovery 11, 909-922 (2012).

Acknowledgments: We are grateful to Calibr's Compound Management and High Throughput Screening groups for their assistance with the project and to the members and leadership of the Macrofilaricide Drug Accelerator, especially Ken Duncan, Richard Elliott, and Scott Mills. We thank Marianne Koschel, Iliana Johannes, Martina Fendler and Venelin Nikolov for their technical support. We thank Russell Wada for pharmacological assistance and expertise. Funding: This work was supported by grants from the Bill \& Melinda Gates Foundation: \#OPP1107194 to Calibr, \#OPP1152825 to GHDDI, \#OPP1134310 to Bonn University, and \#OPP1119043 to JDT, SW and MJT. Author contributions: MST and PGS conceived the project. MAB, KG, PMW, LC, AD, FL, WS, CWM designed, and MAB performed the in vitro and ex vivo experiments. HG, MAB performed bacterial panel experiments. MPH, AH designed and BD, SJF, AE, DS, FL, MPH performed the in vivo experiments using L. sigmodontis. NP, JDT, SW, MT designed and NP, EM, JA, AS, VCC, FFF, PWC, AJN, HMM, BLN, NVG, DNA, TDK performed the in vivo experiments in SCID mice. RKS, RH, JO, BY, JR, XC, HMP designed and performed synthesis and optimization. AKW and SBJ coordinated preclinical 
profiling and pharmacokinetic studies. MVH coordinated the high throughput and compound management groups. WX, KLK outlined the project workflow. MAB wrote, and all authors edited the manuscript. Competing interests: Data and materials availability: All data associated with this study are present in the paper or Supplementary Materials. Results from the primary in vitro screen of the ReFRAME library have been deposited to the reframedb.org data portal. 
Figures and Tables:

Fig. 1. A primary cell-based high-throughput phenotypic screen identifies compounds with potent and selective anti-wolbachial activity. A) Schematic of primary anti-wolbachial screen workflow. B) Representative images from DMSO- and doxycycline-treated wells. One field of view (covering nearly the entire surface of each well) and a zoomed in segment with or without analysis overlays are shown (outline of LDW1 cell nuclei in blue, perimeter of analysis area extending beyond the nucleus in purple, and the identified Wolbachia spots are demarcated with a transparent red mask). In the merged image Wolbachia 16S rRNA FISH signal is colored magenta and DNA signal (DAPI) is colored green. Raw and normalized values (see Materials and Methods) calculated from the images shown in (A) are listed. Scale bar $=100 \mu \mathrm{m}$. C) Heat map images of analysis results from plates ran in triplicate. Normalized \% activity values for Wolbachia signal and cell numbers are indicated according to the scale bar. DMSO-treated wells in column 45 and individual positive control-treated wells (blocks of wells with $12.5 \mu \mathrm{M}$ doxycycline, $0.125 \mu \mathrm{M}$ rifampicin, or $12.5 \mathrm{uM}$ puromycin) in column 46. D) 11 point 1:3 dose response curves of known antibiotics with activity against Wolbachia, including puromycin cell toxicity positive control. E) LDW1 cell number dose response data. F) Mammalian HEK293T cytotoxicity dose response data. Puromycin $\mathrm{CC}_{50}$ is shown. G) Powder reconfirmation results for Bioactive and Diversity libraries and $(\mathbf{H})$ the ReFRAME library, where the $w$ Mel $\mathrm{IC}_{50}$ s of each compound are plotted against their mammalian cytotoxicity (HEK293T CC 50 s). Compounds are color-coded based on library origin. Hit potency and selectivity criteria $\left(\mathrm{IC}_{50}<1 \mu \mathrm{M}, \mathrm{CC}_{50}\right.$ : $_{50}$ $>10$ ) are shown as solid lines and grayed out areas represent values which do not meet these thresholds. Dotted lines represent maximal concentrations tested in dose response studies (e.g. $12.5 \mu \mathrm{M}$ in the anti-wolbachial primary assay).

\section{Fig. 2. Novel small molecules with anti-wolbachial activity possess a narrow anti-bacterial} spectrum. Select powder stocks of compounds identified from Bioactive, Diversity I, and Diversity II libraries that displayed potent anti-wolbachial activity and were not significantly toxic to mammalian cells were tested against a panel of gram-positive and gram-negative bacteria. Bacterial viability inhibition following treatment with $5 \mu \mathrm{M}$ or $20 \mu \mathrm{M}$ of each compound was determined by optical density measurements. 
Fig. 3. Wolbachia populations in B. pahangi adult female worms demonstrate differential susceptibility to anti-wolbachial treatment. The effects of short anti-wolbachial ex vivo treatments on Wolbachia populations within adult female B. pahangi worms were evaluated. Worms were treated ex vivo for three days with doxycycline or anti-wolbachial series lead CBR422 and Wolbachia load was quantified using Wolbachia-specific 16S rRNA FISH and imaging (in distal ovaries) or qRT-PCR (in whole worms or tissues). DAPI (green) and Wolbachia-specific 16S rRNA FISH (white or magenta) staining in ovaries of DMSO (A, D), 1 $\mu \mathrm{M}$ doxycycline $(\mathbf{B}, \mathbf{E})$, and $0.33 \mu \mathrm{M}$ CBR422 (C, F) treated worms. A-C) Images of dissected and stained ovaries acquired using a 10x objective of a confocal microscope. Distal ovaries are indicated with boxes and arrowheads; oviducts and distal uteri are indicated with dashed lines and arrows. Scale bar $=100 \mu \mathrm{m}$. D-F) Images of distal ovaries shown in A-C, acquired using a $63 x$ objective of a confocal microscope. DTC, distal tip cell. Scale bar $=10 \mu \mathrm{m}$. Wolbachia elimination (\%) determined using high content image analysis is indicated for each section. G) Wolbachia quantities in distal ovaries compared to those in whole worms following doxycycline $(\mathrm{n}=3)$ or CBR422 ( $\mathrm{n}=2)$ treatment. H) Wolbachia quantities in distal ovaries compared to those in the entire reproductive tract or body wall tissues following doxycycline treatment $(\mathrm{n}=$ 1). Values for each experiment were normalized to DMSO-treated controls and mean \pm stdev are shown.

Fig. 4. An ex vivo worm-based validation assay rapidly identifies compounds with antifilarial Wolbachia activity. A) Schematic of secondary ex vivo validation screen workflow to assess Wolbachia elimination in B. pahangi adult worm ovaries. Two worms (yielding a maximum of four ovaries) were included in each treatment. B) Characterization of doxycycline activity against Wolbachia within B. pahangi ovaries treated ex vivo. Shaded green region indicates the selected validation threshold of $>75 \%$ Wolbachia elimination following $1 \mu \mathrm{M}$ treatment. Data from $>3$ separate experiments are plotted as mean \pm stdev. C) Representative images from DMSO- and (D) $1 \mu \mathrm{M}$ doxycycline-treated ovaries stained with DAPI (green) and Wolbachia (magenta). Analysis overlays used to quantify Wolbachia-specific signal are represented as a semi-transparent red mask. DTC, the distal tip cell, is indicated with a dashed outline. Scale bar $=10 \mu \mathrm{m}$. Boxes surround areas that are magnified 2.6x to the right of each image; Wolbachia-specific signal (arrow). E) Validation results for potent and selective primary 
screen hits, tested in the assay at $1 \mu \mathrm{M}$. Results are grouped and colored by the library from which each hit originated. Control compounds tested at $1 \mu \mathrm{M}$ : DOX, doxycycline; MIN, minocycline; RIF, rifampicin. Data are represented as mean \pm stdev (1-4 ovaries per treatment). Gray area in the graph represents activities below the set validation threshold. F) $w \mathrm{Bp}$

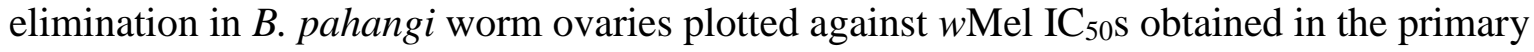
insect cell-based assay. Doxycyline, minocycline, and rifampicin controls are indicated and test compounds are colored by the library from which they originated. Data for quinazolines are indicated as donuts. Gray area in the graph represents activities below secondary assay's validation threshold. G) $w \mathrm{Bp} \mathrm{EC}_{50}$ s obtained for select compounds validated in the secondary worm assay plotted against $w \mathrm{Mel} \mathrm{IC}_{50}$ s obtained in the primary insect cell-based assay.

Compounds are labeled according to library origin. Quinazolines, are indicated as donuts and the structure of the most potent, CBR008, is shown. Coefficient of determination $\left(\mathrm{R}^{2}\right)$ calculated for all compounds is shown in blue (with the associated regression line; dashed blue) and in green for just the quinazolines.Fig. 5. Optimized quinazoline anti-wolbachials demonstrate superior pharmacokinetic profiles. Mice were dosed orally with compounds at indicated amounts. Concentration of each compound in plasma was monitored for at least $24 \mathrm{~h}$. For each compound, $w B p$ EC90s established in the worm-based ex vivo assay are indicated by a red dashed line. Exposure profiles of A) the primary screen hit amide CBR008 and its more potent analog CBR063, B) the oxadiazole series lead CBR422, C) the advanced lead oxadiazole CBR625, D) the optimized series lead oxadiazole CBR417, E) the advanced lead methylpyridine CBR715, and F) the optimized series lead methylpyridine CBR490. CBR008 and CBR063 were formulated in polyethylene glycol $300 / 5 \%$ dextrose in water $(3: 1, \mathrm{v} / \mathrm{v})$; all other compounds were formulated in $40 \%$ (2-Hydroxypropyl)- $\beta$-cyclodextrin. Mean \pm stdev ( $\mathrm{n}=3$ mice) are shown.

Fig. 6. Quinazolines demonstrate anti-wolbachial efficacy in mouse model of $L$. sigmodontis filarial infection. A, B) Advanced anti-wolbachial compounds were assayed in an in vivo model of $L$. sigmodontis filarial infection where mice ( $n=4-6 /$ group) infected with adult $L$. sigmodontis filarial worms (infected by mites carrying L. sigmodontis infectious larvae) are dosed for up to 14 days with a compound of interest. B) Wolbachia load per worm was determined by the ratio of Wolbachia ftsZ gene to that of filarial actin. Vehicle control and a 14day $40 \mathrm{mg} / \mathrm{kg}$ BID doxycycline control were included in each independent experiment. Medians with 95\% CI are shown and median \% elimination is reported (nd, no significant difference; * P- 
value < 0.05; ** P-value < 0.01; *** $\mathrm{P}$-value <0.001; **** $\mathrm{P}$-value <0.0001). DOX, doxycycline; mpk, $\mathrm{mg} / \mathrm{kg}$.

Fig. 7. Quinazoline CBR715 demonstrates anti-wolbachial efficacy in mouse models of $B$. malayi and $\boldsymbol{O}$. ochengi filarial infection. A) Efficacy of CBR715 against Wolbachia in $B$. malayi was assessed in a mouse model of infection where mice ( $n=6 /$ group) are inoculated with infectious L3 larvae of B. malayi. B) Wolbachia content in adult worms determined six weeks after the beginning of treatment is shown. Effect of CBR715 and doxycycline (DOX) control treatments on $\mathbf{C}$ ) the number of $\mathrm{mf}$ circulating in the blood, and $\mathbf{D}$ ) total $B$. malayi worm burden at the end of the in vivo experiments is shown. E) Advanced lead CBR715 efficacy against Wolbachia in O. ocheng $i$ was assessed in a mouse model of infection where mice ( $\mathrm{n}=6 /$ group) are implanted with $O$. ochengi adult male worms. F) Wolbachia content in adult worms determined five weeks after the beginning of treatment. G) Effect of CBR715 and doxycycline (DOX) control treatments on total $O$. ochengi male worms recovered at the end of the in vivo experiments. To assess significance between treatment groups the non-parametric KruskalWallis test with Dunn's multiple comparison test was used. Black lines indicate significant differences between vehicle control and treatment groups and blue lines indicate significant differences between DOX and treatment groups (nd, no significant difference; * P-value $<0.05$; $* *$ P-value $<0.01 ; * * *$ P-value $<0.001 ; * * * *$ P-value $<0.0001)$. 


\begin{tabular}{|c|c|c|c|c|c|c|c|}
\hline & \multirow{2}{*}{$\begin{array}{l}\text { Screen Hit } \\
\text { CBR008 }\end{array}$} & $\begin{array}{c}\text { Failed } \\
\text { Subseries } \\
\text { Lead }\end{array}$ & $\begin{array}{l}\text { Successful } \\
\text { Subseries }\end{array}$ & \multicolumn{2}{|c|}{ Advanced Leads } & \multicolumn{2}{|c|}{ Optimized Leads } \\
\hline & & CBR063 & CBR422 & CBR625 & CBR715 & CBR417 & CBR490 \\
\hline Subseries & amide & amide & oxadiazole & oxadiazole & methylpyridine & oxadiazole & methylpyridine \\
\hline MW & 346.179 & 400.151 & 371.099 & 430.064 & 380.15 & 444.079 & 438.094 \\
\hline \multicolumn{8}{|c|}{ in vitro $\mathrm{HCl}$ cell-based assay: anti-Wolbachia $w$ Mel in $D$. melanogaster LDW1 cells } \\
\hline $\begin{array}{l}\text { anti-Wolbachia wMel } \\
\qquad \mathrm{IC}_{50}(\mathrm{nM})\end{array}$ & 293 & 89 & 7 & 6 & 21 & 24 & 33 \\
\hline $\begin{array}{r}\text { anti-Wolbachia wMel } \\
\qquad \mathrm{IC}_{90}(\mathrm{nM})\end{array}$ & 735 & 200 & 43 & 52 & 182 & 1640 & 283 \\
\hline \multicolumn{8}{|c|}{ ex vivo worm-based assay: anti-Wolbachia $w B p$ in $B$. pahangi ovaries } \\
\hline $\begin{array}{r}\text { anti-Wolbachia wBp } \\
E_{50}(\mathrm{nM})\end{array}$ & 242 & 97 & 26 & 66 & 51 & 356 & $<111$ \\
\hline $\begin{array}{r}\text { anti-Wolbachia wBp } \\
\mathrm{EC}_{90}(\mathrm{nM})\end{array}$ & 799 & 221 & 189 & 346 & 396 & 777 & $<111$ \\
\hline $\begin{array}{l}\% \text { Wolbachia } w \mathrm{Bp} \\
\text { elimination at } 1 \mu \mathrm{M}\end{array}$ & $86.3 \%$ & $98.0 \%$ & $93.5 \%$ & $95.5 \%$ & $94.5 \%$ & $88.0 \%$ & $95.5 \%$ \\
\hline \multicolumn{8}{|c|}{ in vitro mammalian cytotoxicity assay and compound selectivity } \\
\hline HEK293T $\mathrm{CC}_{50}(\mu \mathrm{M})$ & 10.4 & 18.6 & 34.95 & 15.7 & 26.8 & 12.6 & 2.2 \\
\hline HepG2 $\mathrm{CC}_{50}(\mu \mathrm{M})$ & 21.2 & 30.0 & $>40$ & 11.3 & 13.7 & 9.7 & 1.6 \\
\hline HEK293T $\mathrm{CC}_{50} / \mathrm{IC}_{50}$ & 35 & 209 & 5081 & 2735 & 1299 & 525 & 66 \\
\hline HepG2 $\mathrm{CC}_{50} / \mathrm{IC}_{50}$ & 72 & 336 & $>5816$ & 1968 & 663 & 294 & 49 \\
\hline \multicolumn{8}{|c|}{ in vitro L. loa microfilaria selectivity } \\
\hline $\begin{array}{r}\text { L. Ioa microfilaria } \\
\text { motility } I_{50}(\mu \mathrm{M}) \\
\text { (ivermectin control } I C_{50} \\
=11.3 \mu \mathrm{M})\end{array}$ & $n / d$ & $n / d$ & $n / d$ & $n / d$ & $>100$ & 86.9 & 63.6 \\
\hline
\end{tabular}

Table 1. Structures and activities of quinazoline anti-wolbachials. 


\begin{tabular}{|c|c|c|c|c|c|c|c|c|c|c|c|c|c|c|c|c|c|c|c|c|c|}
\hline \multirow{2}{*}{$\begin{array}{l}\text { Dos } \\
(\mathrm{mp}\end{array}$} & & \multicolumn{13}{|c|}{ Days of dosing } & \multirow{2}{*}{$\frac{\text { CBR063 }}{14}$} & \multirow[t]{2}{*}{ CBR422 } & \multicolumn{2}{|c|}{ CBR625 } & \multirow[t]{2}{*}{ CBR715 } & \multirow[t]{2}{*}{ CBR417 } & \multirow[t]{2}{*}{ CBR490 } \\
\hline & & 1 & 2 & 3 & 4 & 5 & 6 & $7^{*}$ & 8 & 9 & 10 & 11 & 12 & 13 & & & & & & & \\
\hline \multirow{12}{*}{ 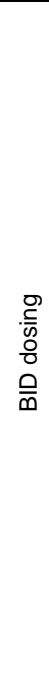 } & 100 & $\bullet$ & .• & $\bullet$ & .• & •• & •• & & & & & & & & & & & & $99.88 \%$ & & \\
\hline & 60 & •• & .• & .• & .• & •• & .• & .• & & & & & & & & & & $99.80 \%$ & & & \\
\hline & 60 & •• & .. & .• & .• & .• & - & & & & & & & & & & & & & & $99.91 \%$ \\
\hline & 50 & •• & .• & •• & .. & .• & .• & .• & •• & .• & •• & .• & .• & •• & •• & $-68.85 \%$ & & & & & \\
\hline & 50 & •• & .• & .• & .• & .• & .• & .• & .• & .• & •• & .• & .• & & & & & & $99.80 \%$ & & \\
\hline & 50 & .. & .. & .• & .. & .. & .• & .. & .• & .• & .• & .. & & & & & $99.59 \%$ & & & & \\
\hline & 50 & .• & .• & •• & .• & .• & .• & •• & & & & & & & & & & & $98.86 \%$ & & \\
\hline & 30 & •• & .• & .• & .• & .• & .• & .• & .• & .• & •• & .• & .• & .• & .• & & & $99.89 \%$ & & & \\
\hline & 30 & •• & .• & .• & .• & •• & .• & .• & .• & .• & •• & .• & & & & & & & & & $99.68 \%$ \\
\hline & 30 & •• & .• & •• & .• & .• & •• & .• & •• & • & & & & & & & & $98.86 \%$ & & & \\
\hline & 30 & •• & .• & .• & .• & .• & .• & .• & & & & & & & & & & $93.20 \%$ & $57.75 \%$ & & \\
\hline & 10 & .• & .. & .• & .. & .. & .• & .• & & & & & & & & & & $66.43 \%$ & & & \\
\hline \multirow{6}{*}{ 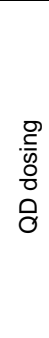 } & 60 & • & - & - & - & - & - & - & & & & & & & & & & $98.95 \%$ & $48.59 \%$ & & $99.72 \%$ \\
\hline & 60 & • & - & - & - & - & - & & & & & & & & & & & & & $99.61 \%$ & \\
\hline & 60 & • & - & - & - & & & & & & & & & & & & & & & $99.96 \%$ & $99.88 \%$ \\
\hline & 30 & • & $\cdot$ & $\cdot$ & $\cdot$ & • & - & - & & & & & & & & & & $42.86 \%$ & & & $99.94 \%$ \\
\hline & 30 & • & - & - & - & & & & & & & & & & & & & & & & $80.73 \%$ \\
\hline & 10 & • & - & - & - & • & - & - & & & & & & & & & & $54.29 \%$ & & & $55.14 \%$ \\
\hline \multirow{2}{*}{$\begin{array}{l}\vec{x} \\
\frac{\vec{v}}{\Phi} \\
3\end{array}$} & 200 & • & & & & & & & & & & & & & & & & & & $99.92 \%$ & $99.88 \%$ \\
\hline & 100 & • & & & & & & & - & & & & & & & & & & & $99.83 \%$ & $99.87 \%$ ** \\
\hline
\end{tabular}

* Desired dosing profile for a macrofilaricide: Oral dose, once daily, up to 7 days or single, intra-muscular injection.

${ }^{* *}$ Mean of two independent experiments

Table 2. Wolbachia elimination from female adult worms achieved following quinazoline treatment in the mouse/L. sigmodontis in vivo model of filarial infection. For ease of interpretation efficacy values are presented in table cells colored on a sliding scale with excellent efficacy (>99\% to 98\% elimination of Wolbachia) in green, medium levels of efficacy (95\% to $80 \%$ elimination) in yellow, and inferior levels in orange ( $70 \%$ to $40 \%$ elimination) and red ( $<30 \%$ elimination). 


\begin{tabular}{|c|c|c|}
\hline & $\begin{array}{c}\text { CBR417 } \\
\text { oxadiazole }\end{array}$ & $\begin{array}{c}\text { CBR490 } \\
\text { methylpyridine }\end{array}$ \\
\hline Molecular Weight (daltons) & 444.079 & 438.094 \\
\hline Solubility (pH 6.8) ( $\mu \mathrm{M})$ & 1 & 1 \\
\hline Melting Point $\left({ }^{\circ} \mathrm{C}\right)$ & $149-153$ & $250-253$ \\
\hline Lipohilicity (cLogP) & 4.44 & 6.18 \\
\hline \multirow{2}{*}{ Plasma Protein Binding } & > $99.9 \%$ (human) & $98.08 \%$ (human) \\
\hline & > $99.9 \%$ (mouse) & $>99.9 \%$ (mouse) \\
\hline \multicolumn{3}{|l|}{ Permeability Caco-2 } \\
\hline Papp A-B $\left(10^{-6} \mathrm{~cm} / \mathrm{s}\right)$ & 2.08 & 0.14 \\
\hline Papp B-A $\left(10^{-6} \mathrm{~cm} / \mathrm{s}\right)$ & 1.79 & 0.26 \\
\hline \multicolumn{3}{|l|}{ Metabolic stability (M / R / D / H) } \\
\hline Extraction ratio (\%) & 52 / 20 / $30 / 41$ & 86 / 35 / 39 / 63 \\
\hline $\mathrm{T}_{1 / 2}(\min )$ & $56 / 145 / 145 / 88$ & 10 / 85 / 100 / 35 \\
\hline $\mathrm{CL}_{\text {int }}(\mu \mathrm{L} / \mathrm{min} / \mathrm{mg})(\mathrm{Rat})$ & $25 / 10 / 10 / 16$ & $137 / 16 / 14$ / 39 \\
\hline \multicolumn{3}{|l|}{ hERG (cardiotoxicity) - manual patch clamp } \\
\hline$\%$ inhibition at $5 \mu \mathrm{M}$ & 20.5 & 27.1 \\
\hline $\mathrm{IC}_{50}(\mu \mathrm{M})$ & 19.5 & 7.1 \\
\hline Cardiac panel safety profiling ${ }^{a}$ & $\begin{array}{l}\text { No significant } \\
\text { inhibition }\end{array}$ & $\begin{array}{c}\text { No significant } \\
\text { inhibition }\end{array}$ \\
\hline Mini-AMES (genotoxicity) & negative & negative \\
\hline Micronucleus induction (mutagenicity) & negative & negative \\
\hline \multicolumn{3}{|l|}{ Cytochrome P-450 isoform $\left(\mathrm{IC}_{50}\right)(\mu \mathrm{M})$} \\
\hline Reversible $1 \mathrm{~A} 2$ & 29.52 & 6.38 \\
\hline Reversible 2C19 & $>50$ & 11.25 \\
\hline Reversible $2 \mathrm{C} 9$ & 45.34 & 6.81 \\
\hline Reversible 2D6 & $>50$ & 13.00 \\
\hline Time-dependent inhibition 3A4 & $>50$ & $>50$ \\
\hline CYP induction, PXR functional assay & $>30$ & $>30$ \\
\hline $\begin{array}{l}\text { Safety pharmacology profiling }{ }^{b} \\
\text { (selected receptors, ion channels, } \\
\text { transporters, kinases, etc.) }\end{array}$ & $\begin{array}{c}4 \text { with }>45 \% \\
\text { binding/inhibition }\end{array}$ & $\begin{array}{c}14 \text { with }>45 \% \\
\text { binding/inhibition }\end{array}$ \\
\hline $\begin{array}{l}\text { P-glycoprotein inhibition, } \\
\text { MDCK-MDR1 } I_{50}(\mu \mathrm{M})\end{array}$ & 6.5 & 1.49 \\
\hline
\end{tabular}


a Table S8

${ }^{b}$ Table S9

Table 3. In vitro ADME and safety profiling data for optimized leads CBR417 and CBR490. 


\section{Bakowski et al. 2018 Supplementary Materials}

\section{Supplementary Materials and Methods:}

\section{Primary in vitro cell-based assay details}

Assay plates were coated with Concanavalin-A to facilitate cell adhesion. The Concanavalin-A solution was dispensed into wells, aspirated after a 10-min incubation, and plates were allowed to dry in a laminar flow hood for at least $2 \mathrm{~h}$, then stored at $4{ }^{\circ} \mathrm{C}$.

For high content analysis, host cell nuclei were identified using DAPI signal and a generous perimeter (radius increased by 5 pixels) was demarcated around this region to define the cell area. Far red (16S rRNA FISH)-stained spots within all identified cells were selected based on their signal intensity above background using the box detection method and fixed thresholding. Analysis outputs included 1) the total number of LDW1 cells per well, and 2) the Wolbachia signal, quantified by the total fluorescence intensity of all 16S rRNA FISH-stained spots within all identified LDW1 cells (averaged per well).

Compounds from five libraries, including one Bioactive (collection of known annotated compounds) and three Diversity libraries (collection of proprietary and commercial compounds with drug-like properties), and the newly-assembled Repurposing, Focused Rescue, and Accelerated Medchem (ReFRAME) (29) were screened at a single concentration of $2.5 \mu \mathrm{M}$ and primary hits showing significant reduction of Wolbachia signal ( $\geq 60$ or $\geq 70 \%$, as indicated) underwent initial triplicate reconfirmation in single point or were directly assayed in 8-point serial dilutions to generate dose response curve data (Table 1). Reconfirmed hits were tested against Wolbachia in 8-point dose response and for cytotoxicity in the mammalian HEK293T cell line. Mammalian cytotoxicity was used to down-select toxic hits, as insect cell toxicity was rare under the screening conditions.

To verify activity and where available, powder stocks of potent $\left(\mathrm{IC}_{50}<1 \mu \mathrm{M}\right)$ and selective $\left(\mathrm{CC}_{50}: \mathrm{IC}_{50}>10\right)$ compounds from the Bioactive and Diversity libraries were re-tested in 11point dose response against Wolbachia and for cytotoxicity in mammalian HEK293T and HepG2 cell lines, with a total of 313 powders tested. The known anti-wolbachials doxycycline and rifampicin were re-discovered in the Bioactive library as potent and selective but were omitted from powder reconfirmation. The ReFRAME library as tested was newly-assembled and in excellent condition (e.g. 54\% of primary hits directly tested in 8-point dose response reconfirmed 
with an $\left.\mathrm{IC}_{50}<1 \mu \mathrm{M}\right)$ and therefore powders of ReFRAME hits were not re-tested in primary assays. Reconfirmed hits from the ReFRAME library have been deposited in the reframedb.org data portal.

\section{Fluorescence in situ hybridization (FISH)}

Two oligonucleotide probes labeled on 5' ends with Quasar 670 (Biosearch Technologies) complementary to Wolbachia $16 \mathrm{~S}$ rRNA were used for FISH labeling experiments. Probe W2 [5'-CTTCTGTGAGTACCGTCATTATC-3'] has been previously described (54) and a new probe Wpan1 [5'-CCCCAGGCGGAATGTTTA-3'] was designed based on E. coli 16S rRNA accessibility data (55). Probes were tested in $w$ Mel-infected LDW1 and tetracycline-cured LDW1 cell line. Each probe is complimentary to the $16 \mathrm{~S}$ rRNA sequences of both $w \mathrm{Mel}$ and $w \mathrm{Bp}$ strains and they were used simultaneously to improve detection. For the in vitro cell-based assay, fixed cells were incubated with FISH hybridization buffer over night at $37{ }^{\circ} \mathrm{C}(50 \%$ deionized formamide, 4× SSC, 10x Denhardts solution, 0.2 M DTT, 0.1\% Tween 20, 0.5 $\mu \mathrm{M}$ $\mathrm{W} 2$, and $0.5 \mu \mathrm{M}$ Wpan 1$)$. Cells were rinsed once and then washed at $42{ }^{\circ} \mathrm{C}$ for $1 \mathrm{~h}$ with $1 \times$ Wash Buffer $(1 \times$ SSC, $10 \mathrm{mM}$ DTT, $0.1 \%$ Tween20), then rinsed and washed for an additional $1 \mathrm{~h}$ with $0.5 \times$ Wash Buffer. For the ex vivo B. pahangi validation assay, dissected ovaries were stained as above, with the following additions to the FISH hybridization buffer to improve sensitivity: $8.25 \%$ dextran sulfate and $0.5 \mu \mathrm{M}$ each unlabeled helper oligos W2H1 [5'TTCCTCACTAAAAGAGCTTT-3'](56), W2H2 [5'-CACGGAGTTAGCCAGGACT-3'](56), and newly-designed Wpan1H1 [5'-ACGCGTTAGCTGTAATAC-3'], Wpan1H2 [5'TAATCTTGCGACCGTAGT-3’].

\section{Mammalian cytotoxicity counter screen}

HepG2 (ATCC) and HEK293T (ATCC) mammalian cell lines were maintained in Dulbecco's Modified Eagle Medium (DMEM, Gibco) with 10\% heat-inactivated HyClone FBS (GE Healthcare Life Sciences), $100 \mathrm{IU}$ penicillin, and $100 \mathrm{mg} / \mathrm{mL}$ streptomycin (Gibco) at $37^{\circ} \mathrm{C}$ with $5 \% \mathrm{CO}_{2}$ in a humidified tissue culture incubator. To assay mammalian toxicity of hit compounds, 750 HepG2 and 375 HEK293T cells/well were seeded in assay media (DMEM, $2 \%$ FBS, $100 \mathrm{IU}$ penicillin, and $100 \mathrm{mg} / \mathrm{mL}$ streptomycin) in 1536-well, white, tissue culture-treated, solid bottom plates (Corning, 9006BC) that contained acoustically transferred compounds in a 
three-fold serial dilution starting at 20 or $40 \mu \mathrm{M}$. After a 72-h incubation, CellTiter-Glo Luminescent Cell Viability Assay (Promega) was used to quantify cell viability as per manufacturer instructions. Luminescence signal was read on the PHERAstar FSX plate reader (BMG Labtech).

\section{Confocal microscopy}

The Leica SP5 confocal microscope was used to image the central plane of ovaries using a 10× air (HCX PL APO CS 10.0x0.40 DRY, Leica Microsystems) or 63× glycerol objective (HCX PL APO CS 63.0x1.30 GLYC $21^{\circ} \mathrm{C}$, Leica Microsystems). Only ovaries where the distal tip cell was positively identified based on cell morphology were imaged and analyzed. For each experiment, parameters of the microscope such as light intensity, exposure time, camera gain, etc., were determined for the DMSO control sample and consistently applied to subsequently imaged specimens. Confocal images were imported and Wolbachia content of each ovary analyzed using Compartmental Analysis in HCS Studio (Thermo Fisher Scientific) as for the cell-based assay.

\section{Relative quantitative RT-PCR}

Adult B. pahangi females were treated as described for the secondary ex vivo assay. After three days of treatment animals were frozen at $-80{ }^{\circ} \mathrm{C}$. TRI Reagent RT (Molecular Research Center Inc.) was added directly to each frozen worm and plates were kept at $-80{ }^{\circ} \mathrm{C}$ until processing. To disrupt animal tissues and bacteria the samples were thawed and processed with the Precellys 24 homogenizer (Bertin Corp) $(2 \times 30$ seconds at 6,500 rpm, 5 second rest between cycles, samples on ice for 5 minutes and disruption cycle repeated) using the VK05 Tough Micro-Organism Lysing Kit ( $0.5 \mathrm{~mm}$ glass beads in $2 \mathrm{~mL}$ tubes). GlycoBlue Coprecipitant (Ambion) was included $(30 \mu \mathrm{g} / \mathrm{mL})$ for improved RNA recovery. RNA extraction was performed according to manufacturer directions. The qScript cDNA SuperMix was used for cDNA preparation from 250 ng of RNA per sample and SsoAdvanced Universal SYBR Green Supermix was used for qRTPCR using the Applied Biosystems ViiA 7 Real-Time PCR System. Primers were designed using the Primer 3 software $(57,58)$. The target gene used to measure Wolbachia load was the $w \mathrm{Bp}$ $w s p$ gene, with primers designed based on the available partial $w \mathrm{Bp} w s p$ cDNA sequence (AY095211.1). The putative B. pahangi splicing factor U2 auxiliary factor $65 \mathrm{kDa}$ subunit (U2AF2) (BPAG_0001183401) gene was chosen as the reference gene for the qRT-PCR assay 
because its $B$. malayi orthologue (Bm1_09760) is similarly expressed across all filarial life cycle stages (59). Likewise, we found that the putative $U 2 A F 2$ is similarly expressed in $B$. pahangi in control and drug-treated animals $\left(\mathrm{C}_{\mathrm{t}}\right.$ value $\% \mathrm{CV}<1.8$ for each experiment $)$ and is expressed at approximately the same $\mathrm{C}_{\mathrm{t}}$ as the target wsp gene in control animals. Relative expression ratios of the target $w s p$ and reference $U 2 A F 2$ gene in control and treated samples were computed correcting for primer efficiency using the Pfaffl method (60).

qRT-PCR Primers

Primers U2_F2 [5'-AGATCGTGATCGACGGAAGT-3'] and U2_R2 [5'-

TTTCAGGTGTACGCTCCTTG-3'] are specific to B. pahangi BPAG_0001183401 (putative U2 auxiliary factor $65 \mathrm{kDa}$ subunit) and span the exon2-exon3 splice junction.

Primers wsp_F1 [5'-TGGTGGTAGTGCATTTGGTT-3'] and wsp_R1 [5'TGGAGTAGGAGCTCGTGAAA-3'] are specific to Wolbachia wBp wsp (partial Wolbachia $w$ Bp surface protein cDNA, AY095211.1).

\section{Data analysis}

Primary in vitro screen and mammalian cytotoxicity counter screen data were uploaded to Genedata Screener, Version 13.0.1-Standard. Data was normalized to DMSO (neutral) and inhibitor control-treated wells (neutral controls minus inhibitors), with $12.5 \mu \mathrm{M}$ doxycycline (Sigma) and $125 \mathrm{nM}$ rifampicin (Sigma) used as positive controls. For the mammalian cytotoxicity counter screen $40 \mu \mathrm{M}$ puromycin (Sigma) was used as the positive control. Replicate data were analyzed using median condensing. Dose response curves were fitted with the four parameter Hill Equation. For the orthogonal ex vivo assay, average Wolbachia spot total intensity per cell data for each ovary were normalized to averaged data obtained from DMSO treated ovaries in the same experiment to determine \% Wolbachia elimination (100 - (mean DMSO - sample)/mean DMSO). Dose response curves were fitted with the variable slope (four parameters) least squares fit using GraphPad Prism. Due to limited data points for each curve (most often three) and data normalization, constraints for bottom $(=0)$ and top $(=100)$ plateau parameters were set. Likewise, qRT-PCR data were normalized to averaged data obtained from DMSO controls in the same experiment to determine \% Wolbachia elimination.

\section{Bacterial panel}


Bacterial inocula were grown at $37^{\circ} \mathrm{C}$ in 384 -well clear bottom plates in Mueller-Hinton II Broth (cation adjusted) in the presence of acoustically transferred compounds of interest, except for Mycobacterium tuberculosis which was cultured in Middlebrook 7H9 medium. After an 18-h incubation ( 5 days for $M$. tuberculosis), the absorbance of the cultures was determined in the Perkin Elmer EnVision microplate reader and \% inhibition of growth relative to vehicle treated control (DMSO) was determined. Bacterial strains used were Bacillus subtilis (ATCC 23857), Enterococcus faecalis (ATCC 29212), Enterococcus faecium (ATCC 35667), Staphylococcus aureus (ATCC 43300), M. tuberculosis (H37Ra; ATCC 25177), Pseudomonas aeruginosa (ATCC 15692), Klebsiella pneumoniae (ATCC 700603), and Acinetobacter baumannii (ATCC 17978). To evaluate the antibacterial activity of CBR417 and CBR490, bacteria were grown at $37^{\circ} \mathrm{C}$ in 1536 -well white solid-bottom plates in Mueller-Hinton II Broth (cation adjusted) in the presence of acoustically transferred compounds in a three-fold serial dilution starting at $40 \mu \mathrm{M}$. After a 24-h incubation, BacTiter-Glo Microbial Cell Viability Assay (Promega) was used to measure bacterial viability as per manufacturer instructions.

Mouse/L. sigmodontis in vivo anti-wolbachial efficacy model of patent filarial infection To assess efficacy of drug candidates in the L. sigmodontis in vivo mouse model, female BALB/c wildtype mice (Janvier) were infected at 6-8 weeks via exposure to Ornithonyssus bacoti mites containing infective L. sigmodontis L3 larvae as previously described (61). The same batch of mites was used to infect all animals within one experiment to allow comparable infections. At 35/36 days post infection (dpi), a time point adult worms have developed in the thoracic cavity, mice were treated by oral gavage with the test compounds at indicated doses and regimens using $40 \%$ Cylclodextrin in water as vehicle and a dosing volume of $10 \mathrm{~mL} / \mathrm{kg}$. As positive control, mice received BID treatments with doxycycline-hyclate (Sigma) dissolved in water at a human bioequivalent dose of $40 \mathrm{mg} / \mathrm{kg}$ for 14 days and negative controls were treated with the drug candidate vehicle. Infected animals were randomly assigned into dose groups $(\mathrm{n}=$ 4-6/group). Experimenters involved in dosing were blinded to treatment group. Necropsies were performed at 65-77 dpi using an overdose of isoflurane (Baxter) and adult worms were isolated from the thoracic cavity and peritoneum and were enumerated. Mice used for L. sigmodontis infection were housed at the animal facility of the Institute for Medical Microbiology, Immunology and Parasitology, University Hospital Bonn, Germany, at a $12 \mathrm{~h} \mathrm{light/dark} \mathrm{cycle} \mathrm{in}$ 
individually ventilated cages with food and water ad libitum. The animal experiments were performed according to the European Union animal welfare guidelines and all protocols had been approved by the Landesamt für Natur, Umwelt und Verbraucherschutz, Cologne, Germany (AZ 84-02.04.2015.A507). Wolbachia depletion in L. sigmodontis female adult worms following compound treatment in vivo was determined as previously described $(36,64)$, where remaining intact female adult worms (up to ten per mouse) were individually assessed for Wolbachia ftsZ and $L$. sigmodontis actin values by qRT-PCR.

\section{SCID mouse in vivo anti-wolbachial efficacy models}

To determine compound efficacy against Wolbachia in O. ochengi and B. malayi, validated murine macrofilaricide drug screening infection models were used, as previously described (10, 11). For the $O$. ochengi model, 12 adult $O$. ochengi males harvested from nodules of naturally infected cattle purchased from local herds near Ngaoundere, Adamawa Province, Cameroon were implanted intraperitoneally into CB.17 SCID mice (purchased from Charles River, UK) (n = 6/group). Treatment commenced $72 \mathrm{~h}$ post implant. Vehicle control was administered for 7 days BID, doxycycline at $25 \mathrm{mg} / \mathrm{kg}$ BID was administered for 28 days, and CBR715 at $50 \mathrm{mg} / \mathrm{kg}$ BID was administered for 7 or 14 days. Sparse PK was taken during treatment, and at the end of the experiment (35 days after treatment was initiated) the adult worm burden, worm motility, and Wolbachia load via qPCR were determined. The washout periods were: 1 week for doxycycline control or 4 and 3 weeks CBR715, for 7- and 14-day treatments, respectively. For the B. malayi model, CB.17 SCID mice ( $\mathrm{n}=5$ /group) were inoculated with 75 B. malayi infectious L3 larvae obtained from female adult Aedes aegypti mosquitoes which were fed with B. malayi microfilariae collected from infected gerbils. Treatment commenced seven weeks post infection. Vehicle control was administered for 5 days BID, doxycycline at $25 \mathrm{mg} / \mathrm{kg}$ BID was administered for 42 days, and CBR715 at $50 \mathrm{mg} / \mathrm{kg}$ BID was administered for 7 or 14 days. At the end of the experiment (13 weeks post infection) the adult worm burden, microfilaria production, and Wolbachia load via qPCR were determined. No washout period existed for the doxycycline control, while washout period for CBR715 was 5 and 4 weeks, for 7- and 14-day treatments, respectively. 


\section{L. loa microfilaria in vitro assay}

L. loa in vitro microfilaria motility drug assays were carried out as previously described (12) with the following amendments. L. loa microfilariae were obtained from splenectomised infected baboons (Papio anubis) infected with the L. loa human strain as previously described (13). The acquisition, care and ethical concerns on the use of baboons as donors of microfilariae have been previously described (13). Ethical and administrative clearances for the use of baboons in this study were obtained from the Ministry of Scientific Research and Innovation of Cameroon (Research permit \#028/MINRESI/B00/C00/C10/C12) and the Animal Care Committee at REFOTDE. Procedures adhered to the NIH Guide for the Care and Use of Laboratory Animals. Purified L. loa were seeded at 30-50 microfilariae per well wells of 96-well plates pre-cultured with a monkey kidney cell line monolayer in DMEM + 10\% FCS (LLC-MK2; a gift from Dr. Simon Townson, Northwick Park Institute for Medical Research). Drug compounds were prepared (blinded) at $100 \mathrm{mM}$ in DMSO before being diluted and added to microfilaria cell cultures in triplicate using a six-point, $0.5 \log$-fold dilution series starting at $100 \mu \mathrm{M}$ in complete medium. The number of immotile (stretched, straight) and motile microfilariae were observed manually in each well using an inverted tissue culture microscope on day 5. Each microfilaria was monitored for a period of approximately 2 seconds to detect any motility. Stretched microfilariae would be more closely monitored for around 5 seconds to confirm no motility especially at anterior or posterior ends over that period before being scored as immotile. The percentage motile microfilariae per well were calculated. Hills Slope $\mathrm{IC}_{50}$ was fitted and calculated using GraphPad Prism v6.0.

\section{Permeability, metabolic stability, protein binding and solubility studies}

Studies were carried out at WuXi AppTec (Shanghai, China). For LC/MS/MS analysis, test and control compounds were quantified using peak area ratio of analyte and internal standard. Caco- 2 cell permeability assay was carried out using a standard protocol. Briefly, Caco-2 cells (ATCC) were seeded onto polyethylene membranes (PET) in 96-well BD Insert plates at $1 \times 10^{5}$ cells $/ \mathrm{cm}^{2}$, and medium was refreshed every 4-5 days until confluent cell monolayer formation (21-28 days post seed). Following addition of compounds, the plate was incubated for $2 \mathrm{~h}$ without shaking and compounds were tested at $2 \mu \mathrm{M}$ bi-directionally in duplicate. Concentrations of test and control compounds in starting solution, donor solution, and receiver 
solution were quantified by LC/MS/MS. The metabolic stability in liver microsomes was determined using the compound depletion approach and quantified by LC/MS/MS. Human, mouse, rat, and dog microsomes were used (BD Gentest). The rate and extent of metabolism was determined by the disappearance of the parent compound, allowing for the determination of in vitro half-life $\left(\mathrm{t}_{1 / 2}\right)$, intrinsic clearance $\left(\mathrm{Cl}_{\text {int }}\right)$ and the extraction ratio $(\mathrm{ER})$ in various species. Protein binding to human and CD-1 mouse plasma was determined using equilibrium dialysis. Compounds were tested at $2 \mu \mathrm{M}$ in triplicate, and concentrations were quantified by LC/MS/MS. Kinetic solubility in $50 \mathrm{mM}$ phosphate buffer $\mathrm{pH} 6.8$ was measured using the shake flask method followed by HPLC-DAD analysis.

\section{Cytochrome $P 450$ analysis}

Quinazolines were subjected to cytochrome P450 inhibition analysis using five different isoforms using standard procedures (human liver microsomes, BD Gentest). Compounds were tested in a 7-point two-fold dilution series starting at $50 \mu \mathrm{M}$. Human PXR induction was evaluated by Puracyp Inc (Carlsbad, CA) in the DPX2 Luciferase cell-based in vitro assay (14) in a 6-point three-fold serial dilution starting at $30 \mu \mathrm{M}$.

\section{P-glycoprotein MDCK-MDR1}

MDR1-MDCK II cells (obtained from Piet Borst at the Netherlands Cancer Institute) were seeded onto polyethylene membranes (PET) in 96-well BD insert systems at $2.3 \times 10^{5} \mathrm{cells} / \mathrm{cm}^{2}$ and grown until confluent cell monolayer formation (4-7 days). Cells were treated with compounds of interest and verapamil (positive inhibitor) in an 8-point three-fold serial dilution starting at $50 \mu \mathrm{M}(100 \mu \mathrm{M}$ for verapamil) together with digoxin at $10 \mu \mathrm{M}$, in duplicate. Permeation of digoxin from apical to basal direction or basal to apical direction was determined over a 150 -minute incubation at $37^{\circ} \mathrm{C}$ and $5 \% \mathrm{CO}_{2}$ with saturated humidity. Digoxin was quantified by LC/MS/MS analysis based on the peak area ratio of analyte and internal standard, and $\mathrm{IC}_{50}$ of the test compounds was calculated by SigmaPlot (V.11).

\section{Pharmacokinetic Studies}

Pharmacokinetic studies were conducted at GVK-Bio (India), Calibr (San Diego, CA), or WuXi (Shanghai, China) in accordance with IACUC guidelines. Male CD-1 fasted mice (three per 
group) were dosed per os (PO) or intravenously (IV) as indicated for each compound and formulation. Plasma concentration of each test article was monitored up to $72 \mathrm{~h}$.

\section{hERG manual patch clamp assay}

Compounds were tested for effect on hERG potassium channels using the whole-cell patch clamp technique with a Multiclamp 700 patch-clamp amplifier (Molecular Devices, USA) at WuXi AppTec (Shanghai, China). CHO cells stably expressing hERG potassium channels from Aviva Biosciences (San Diego, CA) were tested with compounds at five concentrations, in a three-fold serial dilution starting at $30 \mu \mathrm{M}$, and compared to vehicle (negative) control and Amitriptyline (WuXi AppTec, Shanghai, China) (positive) controls. Percentage of control (vehicle) values were calculated in duplicate for each concentration of drug, and data analysis were performed using Clampfit (V10.7, Molecular Deveices) and GraphPad Prism 5.0.

\section{Mini-Ames}

The ability of CBR417 and CBR490 to induce reverse mutations both in the presence and absence of S9 mix at the histidine locus in the genome of four strains of Salmonella typhimurium (TA98, TA100, TA1535, and TA1537) and at the tryptophan locus in the genome of Escherichia coli WP2 uvrA (pKM101) was determined at WuXi AppTec (Shanghai, China). The tested dose levels in the mutagenicity assay with five tester strains in the presence and absence of S9 mix were $1.5,4,10,25,64,160,400$, and $1000 \mu \mathrm{g}$ per well, three wells per dose.

\section{In vitro micronucleus induction}

In vitro microwell micronucleus screening assay in Chinese hamster ovary cells (CHO-WBL, Merck Research Laboratories, USA) was carried out at WuXi AppTec (Shanghai, China). Clastogenicity/aneugenicity was measured by the extent of micronucleus formation with and without exogenous metabolic activation (Aroclor 1254 induced rat liver S9, Molecular Toxicology (Boone, NC)). Cultures of CHO-WBL cells in Microwell 8-well chamber slides (Thermo Fisher Scientific Inc.) were exposed in duplicates to 18 concentrations of CBR417 or CBR490 (444.38 $\mu \mathrm{g} / \mathrm{mL}$ top concentration) as well as to positive (Cyclophosphamide monohydrate, Mitomycin C) and solvent controls. In the S9 activated test system, exposure was for $3 \mathrm{~h}$; in the non-activated test system, treatment was for $3 \mathrm{~h}$ and for $24 \mathrm{~h}$. Cells were fixed, 
stained with acridine orange, and 2000 binucleated cells (1000 binucleated cells/well) were scored for each test and control article concentration.

In vitro safety profiling assays

The Eurofins SafetyScreen44TM Panel (Cerep) and the Cardiac Profiler safety panels were used to assess the off-target effects of CBR417 and CBR490 at a single concentration of $10 \mu \mathrm{M}$. The SafetyScreen44 includes binding and enzymatic inhibition assays performed on targets that have been found associated with adverse drug reactions in vivo (15) and the Cardiac Profiler panel assesses inhibition of eight key cardiac channels in functional cell-based assays. 


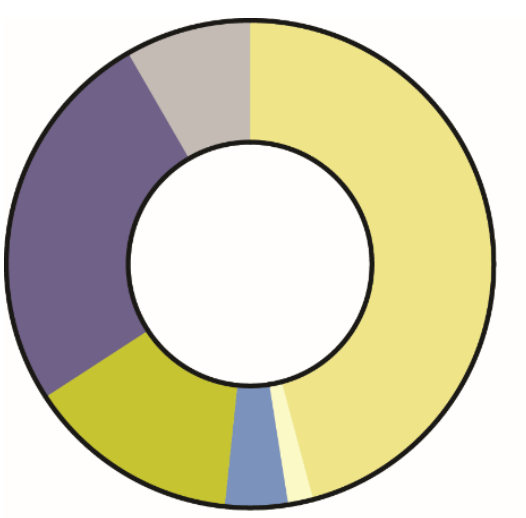

Total $=120$
Antibiotics: $45.8 \%$

tetracyclines

rifamycins

peptide deformylase inhibitors pleuromutilins

fluoroquinolones aminocoumarins macrolides

Antivirals: $1.7 \%$

Antifungals: $4.2 \%$

Antineoplastics: $14.2 \%$

Signal transduction: $25.8 \%$

mTOR

diabetes

liver $\mathrm{X}$ receptor agonist

Other: $8.3 \%$
55

10

9

6

4

4

4

2

2

5

17

31

5

4

2

Supplementary Fig. 1. Classes of known drugs and bioactive molecules identified as potent and selective anti-wolbachial hits in the primary in vitro screen. Duplicates (e.g. retapamulin and ketoconazole, which were identified in both the ReFRAME and Bioactive libraries) were counted only once in this analysis. 

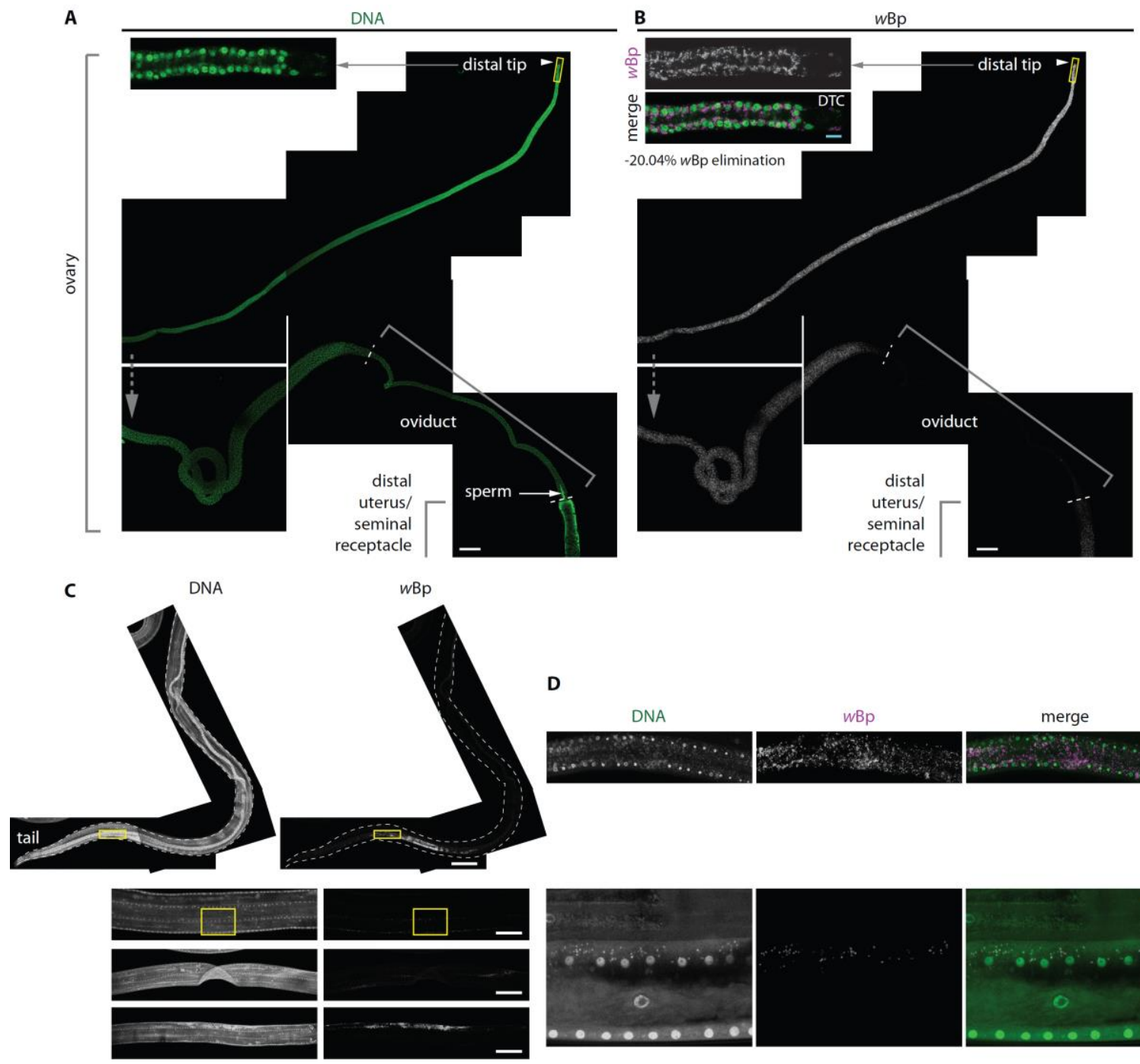

$w B p$

\section{D}

DNA
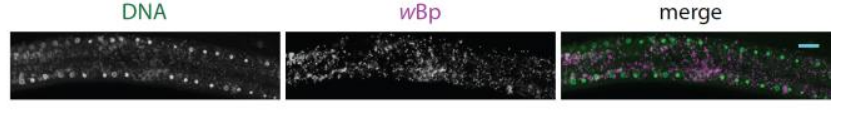

然该
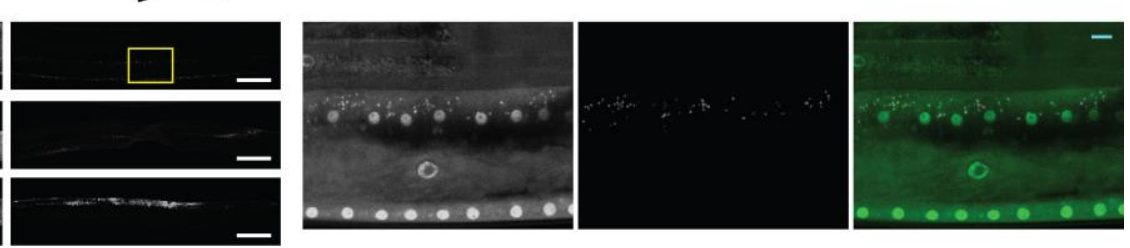

Supplementary Fig. 2. Wolbachia distribution in ovaries and the hypodermis of DMSO-

treated worms. Adult female B. pahangi worms were treated ex vivo with $0.1 \%$ DMSO vehicle control for three days. Worms were fixed and ovaries were dissected away from the body wall sections of the worm. Fragments of each tissue were stained with DAPI (green) and Wolbachiaspecific 16S rRNA FISH (white or magenta) and imaged using a confocal microscope. A composite image of a single worm ovary stained with DAPI (A) and 16S rRNA FISH (B) acquired using a 10x objective is shown. Insets show the distal ovary section (area outlined with a yellow box) acquired using a 63x objective. Oviduct, largely devoid of Wolbachia is indicated. DTC, distal tip cell. Variable Wolbachia distribution in representative sections of the hypodermis imaged using a 10x (C) or 63x (D) objective are shown. Magnified areas shown in $\mathbf{D}$ are outlined in $\mathbf{C}$ with yellow boxes. Scale bars (white) $=100 \mu \mathrm{m}$. Scale bars (cyan) $=10 \mu \mathrm{m}$. 


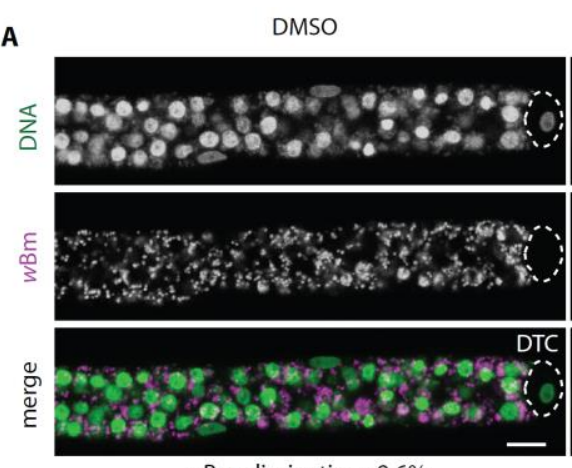

$w B m$ elimination:-9.6\%

CBR422 $1 \mu \mathrm{M}$

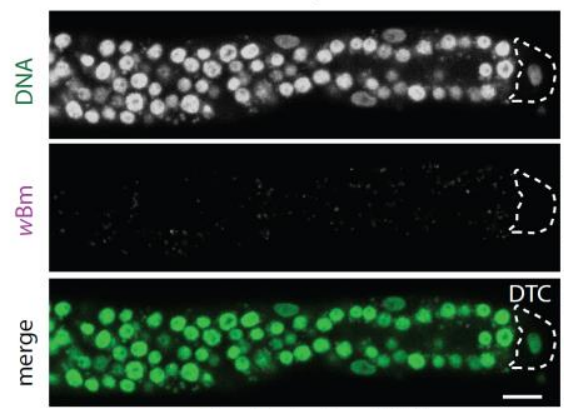

wBm elimination: $95.8 \%$

Doxycycline $1 \mu \mathrm{M}$

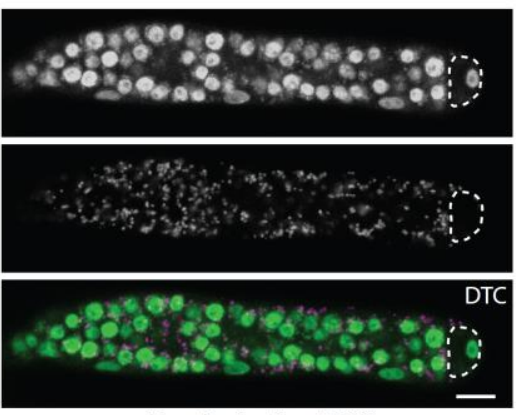

wBm elimination: $55.3 \%$

Tylosin $1 \mu \mathrm{M}$

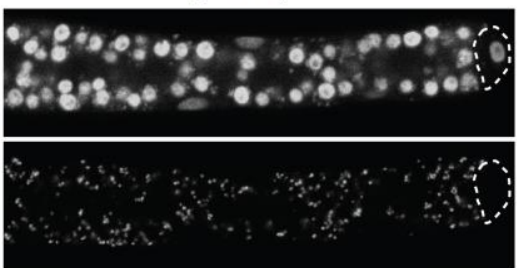

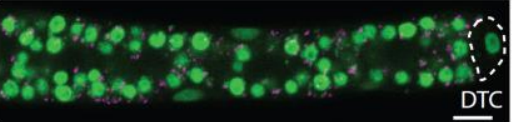

wBm elimination: $62.0 \%$

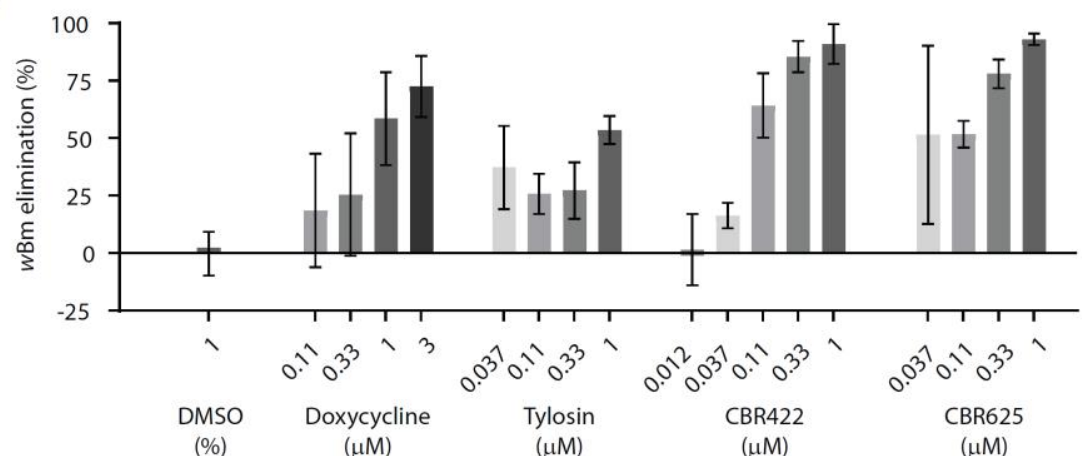

C

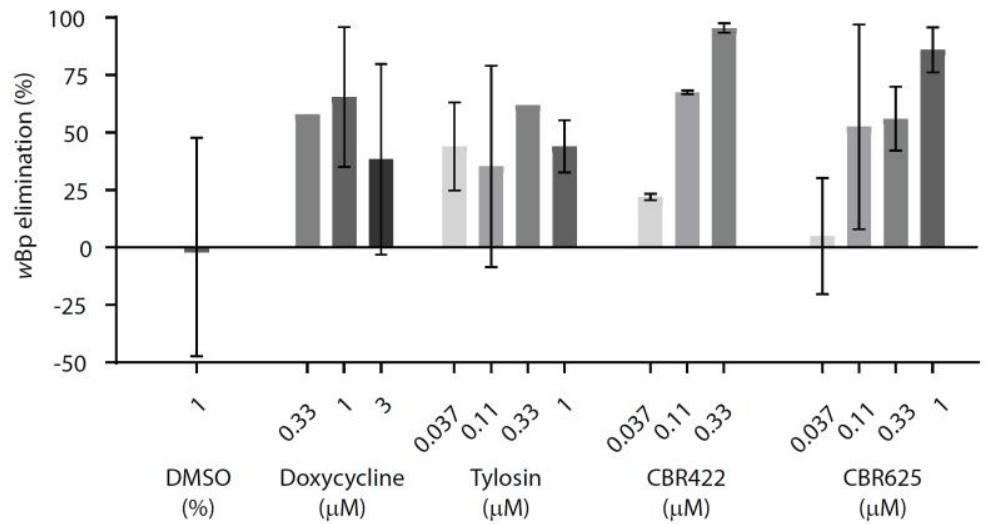

CBR625 $1 \mu \mathrm{M}$

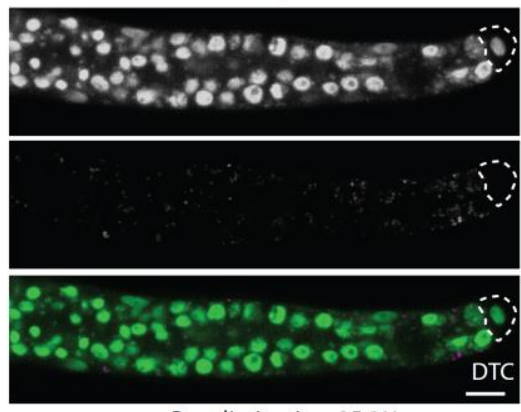

$w$ Bm elimination: $95.2 \%$ 
Supplementary Fig. 3. Wolbachia elimination from B. malayi worm ovaries ex vivo. As in the secondary validation screen, B. pahangi and B. malayi adult female worms were maintained ex vivo in the presence of compounds of interest. After three days, the worms were frozen, fixed, and dissected to isolate the ovaries. The ovaries were stained for Wolbachia 16S rRNA and total DNA, and their distal tips imaged using a confocal microscope with a 63x objective. The images were analyzed for the specific Wolbachia signal using HCI analysis software. Two worms (yielding a maximum of four ovaries) were included in each treatment except for the DMSO treatment where four worms were included. A) Representative images from neutral control (DMSO-treated), positive control ( $1 \mu \mathrm{M}$ doxycycline-treated), $1 \mu \mathrm{M}$ CBR422, $1 \mu \mathrm{M}$ tylosin, and $1 \mu \mathrm{M}$ mCEB6625-treated B. malayi ovaries stained with DAPI (green) and Wolbachia (magenta). DTC, the distal tip cell, is indicated with a dashed outline. Scale bar $=10 \mu \mathrm{m}$. B) Compound activity against $w B m$ within $B$. malayi ovaries treated ex vivo. Data from one experiment are plotted as mean \pm stdev. C) Compound activity against $w B p$ within $B$. pahangi ovaries treated ex vivo determined at the same time as for B. malayi. Data from one experiment are plotted as mean \pm stdev. D) $\mathrm{EC}_{50}$ values estimated from data in $\mathrm{B}$ and $\mathrm{C}$. 

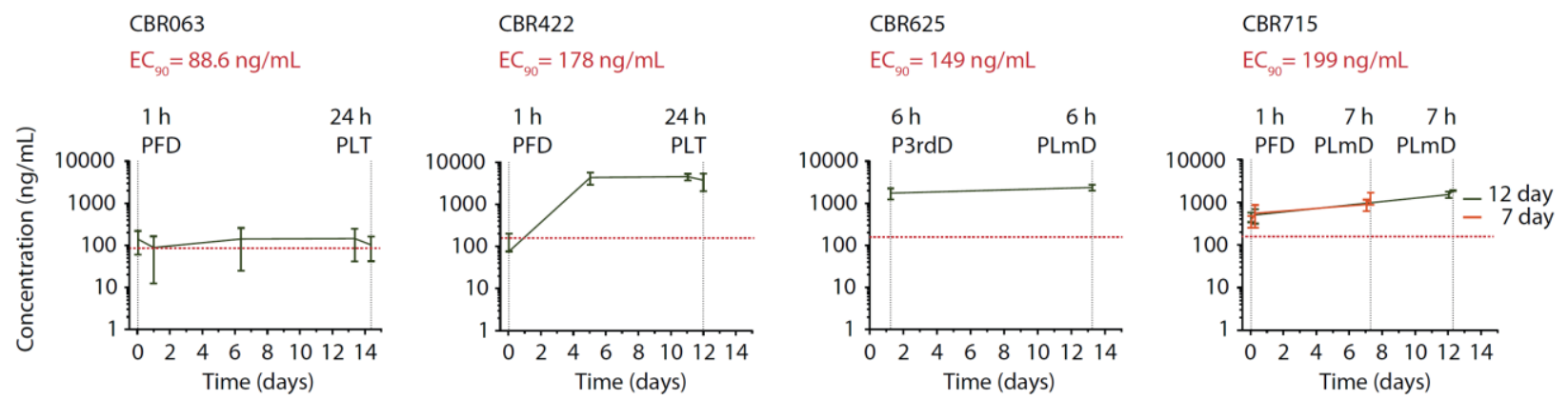

\section{Supplementary Fig. 4. Sparse pharmacokinetic profiles of quinazoline anti-wolbachials}

during efficacy studies. Limited exposures were determined during rodent models of filarial infection. Sparse pharmacokinetic profiles for efficacy studies performed in the L. sigmodontis mouse model of infection. Formulations and BID doses are as in figure 6B. PFD, post first dose; PLT, post last dose; P3rdD, post third dose; PLmD, post last morning dose. 

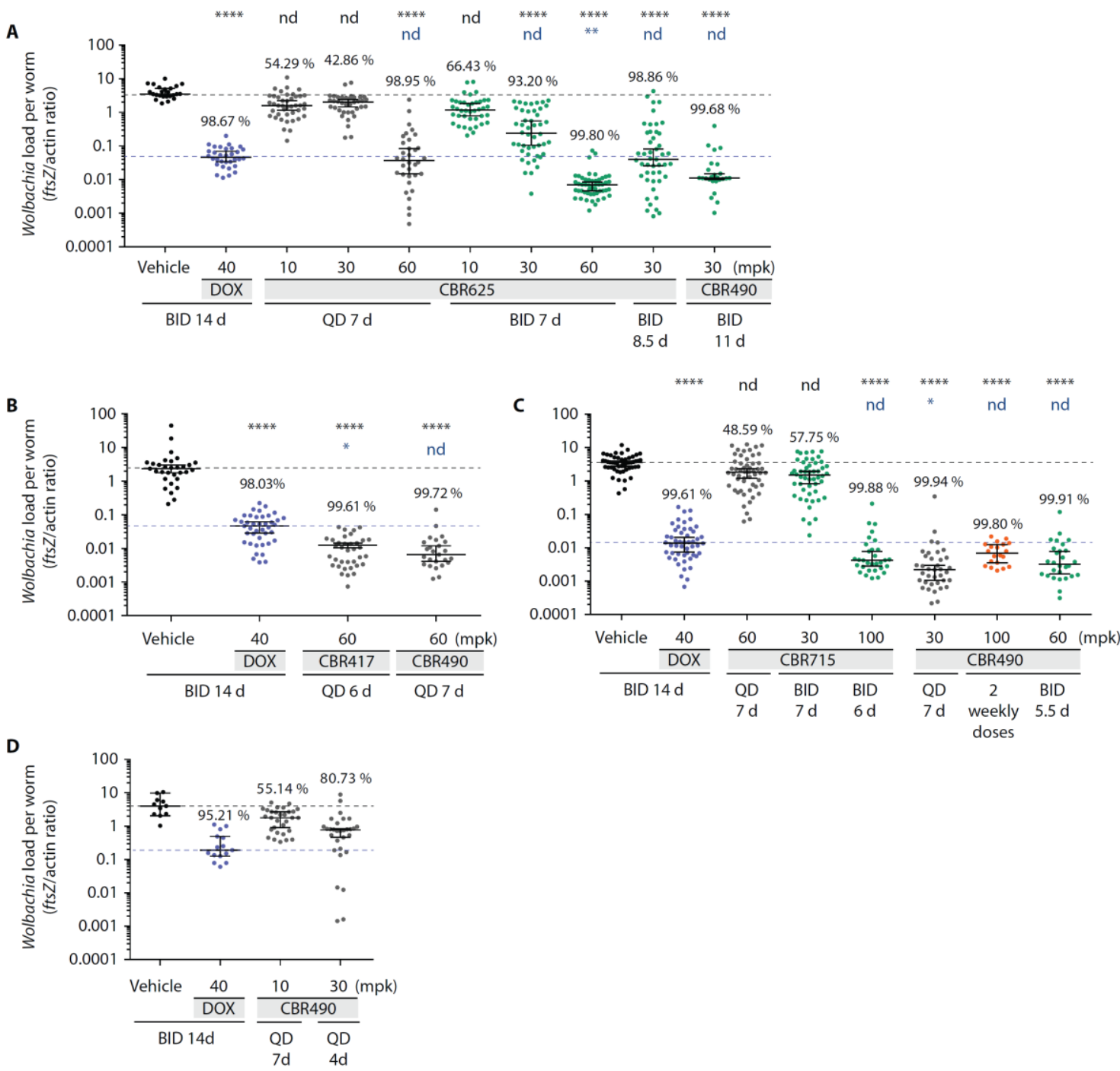

\section{Supplementary Fig. 5. Wolbachia elimination following quinazoline treatment in the}

mouse/L. sigmodontis in vivo model of filarial infection. Data, for which median \% Wolbachia elimination is reported in Table 2 of main text is shown here. Wolbachia quantities per worm are determined by the ratio of Wolbachia ftsZ gene to that of filarial actin. Vehicle control (black puncta and dashed lines for median values) and a 14-day $40 \mathrm{mg} / \mathrm{kg}$ BID doxycycline control (blue puncta and dashed lines for median values) is included for each experiment (A-D). Note that data in (B) are from the same experiment as shown in figure 6B testing efficacy of CBR715, and data in (D) are from the same experiment as shown in figure 6B testing efficacy of CBR417 and CBR490. These shared controls with data reported in main figure. Medians with 95\% CI are shown (nd, no significant difference; * P-value < 0.05; ** P-value <0.01; **** P-value < 0.0001). DOX, doxycycline; $\mathrm{mpk}, \mathrm{mg} / \mathrm{kg}$. 

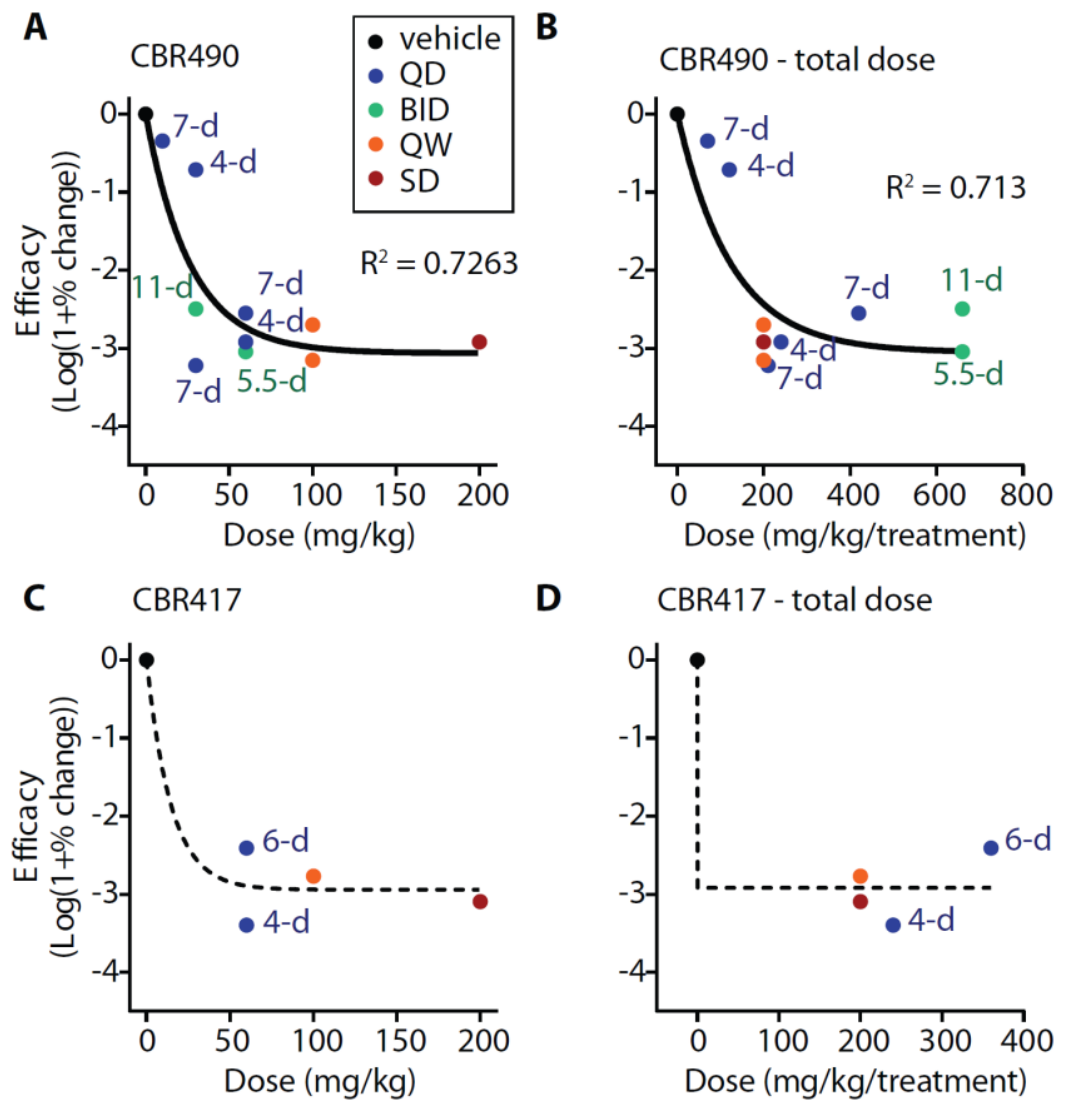

\section{Supplementary Fig. 6. CBR417 and CBR490 dose-response relationship based on}

Wolbachia elimination in mouse/L. sigmodontis efficacy studies. Efficacy, expressed as Log (1+\% change from vehicle control in Wolbachia levels), was plotted against dose (mg/kg) and total dose (mg/kg/day) for CBR490 (A, B) and CBR417 (C, D). An exponential one-phase decay analysis was applied to generate the dose-response relationship models, however sufficient data was unavailable to generate unambiguous curves for CBR417 studies. 


\begin{tabular}{|c|c|c|c|c|c|c|}
\hline $\begin{array}{l}\text { Library } \\
\text { (primary screen format) }\end{array}$ & $\begin{array}{l}\text { Bioactives } \\
\qquad(384 w)\end{array}$ & $\begin{array}{l}\text { Diversity I } \\
\text { (1536w) }\end{array}$ & $\begin{array}{l}\text { Diversity II } \\
\qquad(384 w)\end{array}$ & $\begin{array}{l}\text { Diversity III } \\
\qquad(1536 \mathrm{w})\end{array}$ & $\begin{array}{l}\text { ReFRAME } \\
\text { (1536w) }\end{array}$ & Total \\
\hline Compounds screened [2.5 $\left.\mu \mathrm{M}^{a}\right]$ & 10,673 & 86,844 & 94,697 & 95,337 & 12817 & 300,368 \\
\hline Primary hits ${ }^{b}$ & 324 & 424 & 807 & 199 & 360 & 2,114 \\
\hline Average Z' & 0.6253 & 0.5224 & 0.539 & 0.4058 & 0.5730 & 0.5331 \\
\hline Hit rate & $3.04 \%$ & $0.50 \%$ & $0.85 \%$ & $0.21 \%$ & $2.81 \%$ & $0.70 \%$ \\
\hline Reconfirmation rate & $79.3 \%$ & $\begin{array}{l}\text { (straight } \\
\text { into } D R \text { ) }\end{array}$ & $48.3 \%$ & $33.2 \%$ & $\begin{array}{l}\text { (straight } \\
\text { into } D R \text { ) }\end{array}$ & \\
\hline Tested in dose response (DR) & 214 & 448 & 390 & 78 & 357 & 1,487 \\
\hline $\mathrm{IC}_{50}<1 \mu \mathrm{M}$ & $\begin{array}{c}133 \\
(<1.1 \mu \mathrm{M})\end{array}$ & 143 & $\begin{array}{c}171 \\
(<1.1 \mu \mathrm{M})\end{array}$ & $\begin{array}{c}43 \\
\left(60 \mathrm{IC}_{50}<2 \mu \mathrm{M}\right)\end{array}$ & 194 & 684 \\
\hline $\mathrm{IC}_{50}<1 \mu \mathrm{M}, \mathrm{CC}_{50}: \mathrm{IC} \mathrm{C}_{50}>10$ & 60 & 128 & 162 & $\begin{array}{c}30 \\
\left(39 \mathrm{IC}_{50}<2 \mu \mathrm{M}\right)\end{array}$ & 101 & 481 \\
\hline Tested as powders & $46^{c}$ & 116 & 161 & 36 & $n / a$ & 313 \\
\hline $\begin{array}{l}\text { Powders reconfirmed (1536w) } \\
\mathrm{IC}_{50}<1 \mu \mathrm{M}\end{array}$ & 38 & 35 & 115 & 30 & $n / a$ & 218 \\
\hline Powder reconfirmation rate & $83 \%$ & $30 \%$ & $71 \%$ & $83 \%$ & $n / a$ & $70 \%$ \\
\hline $\begin{array}{l}\text { Potent and selective }\left(\mathrm{IC}_{50}<\right. \\
\left.1 \mu \mathrm{M}, \mathrm{CC}_{50}: \mathrm{IC}_{50}>10\right)\end{array}$ & 33 & 24 & 108 & 30 & $n / a$ & 296 \\
\hline $\begin{array}{l}\text { Filtered for ex vivo validation } \\
\text { screen }^{d}\end{array}$ & 20 & 21 & 53 & 9 & 34 & 137 \\
\hline $\begin{array}{l}\text { Validated ( }>75 \% w B p \\
\text { elimination in ovaries) }\end{array}$ & 2 & 4 & 19 & 3 & 4 & 32 \\
\hline Validation rate & $10.0 \%$ & $19.0 \%$ & $35.8 \%$ & $33.3 \%$ & $11.8 \%$ & $23.4 \%$ \\
\hline Hits of interest & PQ 401 & & $\begin{array}{l}\text { quinazolines: } \\
\text { CBR008 }\end{array}$ & & $\begin{array}{c}\text { tylosin } \\
\text { ketoconazole }\end{array}$ & \\
\hline
\end{tabular}

${ }^{a}$ A fraction of Diversity III library was screened at $1.25 \mu \mathrm{M}$.

${ }^{b}$ Primary hit thresholds: Bioactives, Diversity II, Diversity III, and ReFRAME: $\geq 60 \%$ inhibition. Diversity I: $\geq 70 \%$ inhibition.

$c 14$ compounds were omitted due to overt toxicity in mammalian cells (ten compounds had $\left.\mathrm{CC}_{50} \mathrm{~s}<1 \mu \mathrm{M}\right)$ or duplication $(2 \mathrm{x}$ doxycycline and $2 x$ novobiocin were removed)

${ }^{d}$ Hits filtered for validation based on activity and structure relationships.

n/a: not applicable

Table S1. Primary and validation screen statistics. 


\begin{tabular}{lcc} 
& \multicolumn{2}{c}{$\mathrm{IC}_{50}(\mu \mathrm{M})$} \\
\cline { 2 - 3 } & $\mathrm{CBR} 417$ & $\mathrm{CBR} 490$ \\
\hline Acinetobacter baumannii & $>40$ & $>40$ \\
Bacillus subtilis & $>40$ & $>40$ \\
Enterococcus faecalis & $>40$ & $>40$ \\
Enterococcus faecium & $>40$ & $>40$ \\
Escherichia coli & $>40$ & $>40$ \\
Klebsiella pneumoniae & $>40$ & $>40$ \\
Pseudomonas aeruginosa & $>40$ & $>40$ \\
Staphylococcus aureus & 10.67 & $>40$
\end{tabular}

Table S4. Activity of optimized anti-wolbachial leads against a panel of gram-positive and gram-negative bacteria. 


\begin{tabular}{|c|c|c|c|c|c|}
\hline & Screen Hit & $\begin{array}{c}\text { Failed } \\
\text { Subseries }\end{array}$ & $\begin{array}{l}\text { Successful } \\
\text { Subseries }\end{array}$ & \multicolumn{2}{|c|}{ Advanced Leads } \\
\hline & CBR008 & CBR063 & CBR422 & CBR625 & CBR715 \\
\hline subseries & amide & amide & oxadiazole & oxadiazole & methylpyridine \\
\hline Kinetic Solubility $(\mu \mathrm{M})$ & 1 & 1 & 1 & 2.76 & 1.11 \\
\hline Melting Point $\left({ }^{\circ} \mathrm{C}\right)$ & $n / d$ & $n / d$ & $n / d$ & $155-157$ & 243 \\
\hline cLogP & 5.68 & 6.1 & 4.68 & 3.94 & 5.88 \\
\hline LipE & 0.85 & 0.95 & 3.48 & 4.30 & 1.81 \\
\hline Plasma Protein Binding & $\begin{array}{l}99.43 \% \\
\text { (mouse) }\end{array}$ & $\begin{array}{l}99.72 \% \\
\text { (mouse) }\end{array}$ & $\begin{array}{c}99.345 \% \\
\text { (mouse) }\end{array}$ & $\begin{array}{l}99.51 \% \\
\text { (mouse) }\end{array}$ & $\begin{array}{l}99.98 \% \\
\text { (human) } \\
98.53 \% \\
\text { (mouse) }\end{array}$ \\
\hline Metabolic stability & & & & & \\
\hline $\mathrm{CL}_{\text {int }}(\mu \mathrm{L} / \mathrm{min} / \mathrm{mg})$ (Mouse) & 438.03 & 129.36 & 13.379 & 70.3 & 67.2 \\
\hline $\mathrm{CL}_{\text {int }}(\mu \mathrm{L} / \mathrm{min} / \mathrm{mg})$ (Human) & $n / d$ & $n / d$ & 27.51 & 39.06 & 34.339 \\
\hline $\mathrm{CL}_{\text {int }}(\mu \mathrm{L} / \mathrm{min} / \mathrm{mg})(\mathrm{Dog})$ & $n / d$ & $n / d$ & $n / d$ & 9.6 & 28.52 \\
\hline $\mathrm{CL}_{\text {int }}(\mu \mathrm{L} / \mathrm{min} / \mathrm{mg})$ (Rat) & $n / d$ & $n / d$ & $n / d$ & 9.6 & 30.505 \\
\hline ER (Mouse) & 0.95 & 0.85 & 0.37 & 0.756 & 0.747 \\
\hline ER (Human) & $n / d$ & $n / d$ & 0.5446 & 0.629 & 0.599 \\
\hline ER (Rat) & $n / d$ & $n / d$ & $n / d$ & 0.2 & 0.499 \\
\hline ER (Dog) & $n / d$ & $n / d$ & $n / d$ & 0.3 & 0.571 \\
\hline \multicolumn{6}{|l|}{$\begin{array}{r}\text { Cytochrome P-450 isoform } \\
\text { inhibition }\left(\mathrm{IC}_{50}\right)(\mu \mathrm{M})\end{array}$} \\
\hline CYP 1 A2 & $n / d$ & 3.00 & 0.33 & 5.14 & 1.09 \\
\hline CYP 2C19 & $n / d$ & $>50$ & 7.51 & 8.61 & 23.26 \\
\hline CYP 2C9 & $n / d$ & $>50$ & 7.11 & 17.81 & 17.86 \\
\hline CYP 2D6 & $n / d$ & $>50$ & 27.73 & 32.77 & $>50$ \\
\hline CYP 3A4 & $n / d$ & $>50$ & 9.68 & $>50$ & $>50$ \\
\hline \multicolumn{6}{|l|}{$\begin{array}{r}\text { hERG inhibition } \\
\text { (cardiotoxicity) }\end{array}$} \\
\hline$\%$ inhibition at $5 \mu \mathrm{M}$ & $n / d$ & 6.59 & $n / d$ & 46.24 & 9.6 \\
\hline $\mathrm{IC}_{50}(\mu \mathrm{M})$ & $n / d$ & $n / d$ & 11.81 & 5.03 & $n / d$ \\
\hline \multicolumn{6}{|l|}{ Permeability Caco-2 } \\
\hline Papp A-B $\left(10^{-6} \mathrm{~cm} / \mathrm{s}\right)$ & $n / d$ & 0.047 & 2.98 & $n / d$ & 0.41 \\
\hline Papp B-A $\left(10^{-6} \mathrm{~cm} / \mathrm{s}\right)$ & $n / d$ & 0.086 & 2.62 & $n / d$ & 0.96 \\
\hline
\end{tabular}

Table S6. ADME properties of quinazoline anti-wolbachials. 


\section{Supplementary tables provided as Excel files:}

Table S2. Anti-wolbachial activities of known drugs and bioactive molecules identified as potent and selective hits in the primary in vitro screen (powders and ReFRAME compounds).

Table S3. Antibacterial activities of primary screen hits from Bioactive, Diversity I and Diversity II libraries.

Table S5. Activities of screening hits validated ex vivo (all except ReFRAME compounds are powders).

Table S7. Pharmacokinetic properties of anti-wolbachial quinazolines.

Table S8. Cardiac Panel Study Results for CBR417 and CBR490.

Table S9. Safety Pharmacology Profiling Study Results for CBR417 and CBR490.

Data file S1. Primary data. 